\title{
ANALYSIS OF RF SHEATH INTERACTIONS IN TFTR
}

\author{
D. A. D’Ippolito ${ }^{\dagger}$ and J. R. Myra \\ Lodestar Research Corporation, Boulder, Colorado \\ J. H. Rogers, K. W. Hill, J. C. Hosea, R. Majeski, G. Schilling and J. R. Wilson \\ Princeton Plasma Physics Laboratory, Princeton, New Jersey \\ G. R. Hanson, A. C. England, and J. B. Wilgen \\ Oak Ridge National Laboratory, Oak Ridge, Tennessee
}

\begin{abstract}
New theoretical and experimental tools are applied to the analysis of ICRF antenna-edge plasma interactions in the Tokamak Fusion Test Reactor (TFTR) tokamak. A new numerical method for computing the three-dimensional rf sheath voltage distribution is used, and the quantitative predictions of rf sheath theory are compared with measurements of the edge density profile obtained by microwave reflectometry and with titanium impurity concentration data. It is shown that the local density depletion at the antenna is consistent with density pump-out by strong $\mathbf{E} \times \mathbf{B}$ convection into the Faraday screen. Modeling of the Faraday screen impurity influx shows that the calculated Ti impurity concentration based on this direct influx agrees with the measured concentration for $\pi$ phasing. It is also shown that screening of impurity neutrals by ionization in the SOL is a large effect and increases with rf power. At high power over many shots, a fraction of the metal impurities migrates around the machine and is deposited on the limiters, providing a secondary source of titanium. The data shows that the central $\mathrm{Ti}$ concentration is strongly dependent on antenna phasing. Possible explanations for this phasing dependence are discussed.
\end{abstract}

†email: dasd@lodestar.com

Subject classification: G2, Te; G5, Tt; I1, Tm 


\section{INTRODUCTION}

The ability of Ion Cyclotron Range of Frequency (ICRF) antenna systems to heat fusion plasmas in a wide range of plasma conditions is by now well established, and a substantial research program is underway to employ ICRF systems for driving steady state current in tokamaks. Experiments have shown that ICRF antenna systems can be designed to be robust and effective if certain critical ICRF-edge plasma interactions are controlled. Under adverse conditions, ICRF systems can lead to increased impurity injection from antennas and nearby limiters, uncontrolled density rise, modifications of the scrape-off-layer (SOL) density and temperature profiles, excessive power dissipation at the edge and parasitic antenna loading, and "hot spots" and arcing on the antenna. These effects can degrade the central plasma, reduce the heating efficiency, and damage the antenna and limiters. Control of these deleterious interactions imposes limitations on the antenna voltage and the relative phasing of the current straps, thus limiting the power and $\mathrm{k}_{\|}$of the launched fast wave $(\mathrm{FW})$. Future developments in ICRF physics and technology, such as optimized FW current drive systems and the development of compact, high-power ICRF launchers, demand a good understanding of ICRF-edge interactions.

A great deal of recent progress has been made in understanding and control of these interactions. An extensive summary of experimental and theoretical work in this area can be found in conference proceedings [1] and in recent review articles [2,3]. It was proposed that many of the deleterious ICRF-edge interactions are caused by the formation of radiofrequency (rf) sheaths [4-9]. Theoretical work, modeling, and experiments on several tokamaks have established the importance of rf sheaths and have studied their effects [4-22]. The present paper discusses an analysis of rf sheath effects during FW heating and current drive on the TFTR experiment. This work has led to further advances in the understanding of rf sheath phenomena and in our ability to quantitatively analyze the resulting edge interactions. 
The physics of rf sheaths and the terminology used in the present paper can be summarized briefly as follows. The formation of rf sheaths is induced by the action of an rf field with a substantial component $\mathrm{E}_{\|}$parallel to the equilibrium magnetic field. In the vicinity of a material boundary, electrons are accelerated out of the plasma by $\mathrm{E}_{\|}$faster than the heavier ions, and a confining DC ("rectified") sheath potential $\phi$ develops to confine electrons and restore equilibrium quasineutrality [4-9]. This potential is nearly constant along the equilibrium magnetic field but varies rapidly across it. The spatial variation of $\phi$ drives rapid $\mathbf{E} \times \mathbf{B}$ convection $[12,20,23,24]$ which increases the flux of plasma to the antenna and thereby enhances the strength of the antenna-plasma interactions. The DC electric fields in the SOL and the convective fluxes have been measured with probes in several experiments (e.g. see the discussion in Ref. [3]). During high power operation the induced sheath potential is large $\left(\phi>>3 \mathrm{~T}_{\mathrm{e}}\right)$ and the energy Ze $\phi$ of ions accelerated in the rf sheaths can be in the range $0.5-2 \mathrm{keV}$ where the sputtering yield of metal impurities peaks, leading to greatly enhanced impurity influxes from the antenna [9-11], and limiters or other nearby surfaces $[8,13]$. The energetic ion flux out of the plasma also produces a parasitic power loss, which we refer to as sheath power dissipation $[11,21]$. Under extreme conditions the sheath power dissipation can significantly reduce the heating efficiency [11] and cause overheating of the antenna structure $[18,22]$. At very low $(\sim \mathrm{kW})$ power levels, the sheath power dissipation dominates the antenna loading (when multipactoring is not a factor); in this regime the low-power loading can be used as a non-perturbative diagnostic of sheath formation [21].

There are several possible mechanisms by which FW antennas can produce a large $\mathrm{E}_{\|}$component and thereby induce sheath formation. First, an $\mathrm{E}_{\|}$component will be created in the near field of the antenna by a mismatch between the orientations of the equilibrium magnetic field and the antenna structure [4-11]. This is discussed in more detail in Sec. 3. We refer to this mechanism as "near-field" sheath formation, and it is the dominant process during heating and current drive scenarios with good single pass 
absorption. When the single pass absorption is poor and there is a significant amount of unabsorbed or reflected wave energy near the plasma boundary, "far-field" sheaths can form around the machine. The $\mathrm{E}_{\|}$driving these sheaths can result from the formation of a surface mode $[8,25]$ or from the mismatch between the flux surface and the external wall [13].

One of the important "signatures" of rf sheath phenomena is their dependence on the relative toroidal phasing of the current straps in the antenna. In general, all of the sheath formation mechanisms just described have a phasing dependence, and the origin of this dependence is different for each mechanism. For near-field sheaths, the phasing dependence is caused by cancellation of the $\mathrm{E}_{\|}$fields (or equivalently the rf magnetic flux) produced by adjacent current straps; this cancellation is largest in anti-symmetric phasing where the phase difference between adjacent straps is $\pi$. For far-field sheaths or other rf processes at the walls and limiters, the phasing dependence arises from the $\mathrm{k}_{\|}$ dependence of the single-pass absorption and of the spatial location of the wave cut-offs. In optimizing the ICRF coupling on an experiment, it is important to identify which mechanism is responsible for the observed phasing dependence. We return to this subject in Sec. 5.

We have applied the rf sheath model to an analysis of TFTR edge physics data with the goals of (i) testing and extending the physics basis of the model, and (ii) identifying the mechanisms responsible for changes in the SOL density profiles and for the core metal impurity concentration in the experiment. This paper attempts to give a unified treatment of all aspects of the TFTR sheath analysis. In the course of this work, new theoretical and modeling tools were developed, and data from a new experimental diagnostic (microwave reflectometry [26]) provided a stringent test of the rf sheath model. The TFTR analysis reported here includes: 
(a) a quantitative calculation of the sheath voltage distribution for the realistic three-dimensional antenna geometry and electromagnetic field pattern using the new ANSAT sheath analysis code [19];

(b) a comparison of a new model [27] of sheath-induced convection [12] with direct measurements of the density profile in front of the antenna using microwave reflectometry [26];

(c) use of the measured density profiles to improve the accuracy of the calculations of Faraday screen (FS) impurity generation and of impurity screening by ionization in the SOL.

The modeling results presented here indicate that the earlier picture of $\mathrm{rf}$ sheath physics [9-13], developed to model edge interactions on the Joint European Torus (JET), gives good agreement with the TFTR ICRF-edge physics data when suitably extended. The ANSAT analysis of Sec. 3 indicates the importance of a new class of screen-limiter sheaths for antennas with slotted sidewalls. The generalized convective cell analysis of Sec. 4 reconciles the experimental measurements of rf density depression at the antenna on various tokamaks $[20,26,28]$ with other experimental evidence that the plasma flux to the antenna increases with rf power $[10,12]$. Finally, the FS impurity analysis in Sec. 5 for mode conversion current drive experiments shows reasonable agreement with the data and demonstrates the importance of SOL screening in reducing the direct influx of impurities from the antenna. The conclusions of this analysis are consistent with the results of an earlier analysis of nickel impurity data on JET [10].

The remainder of this paper is organized as follows. The TFTR experimental arrangement and relevant experimental results on ICRF-edge plasma interactions are summarized in Sec. 2. The quantitative analysis of TFTR antenna sheaths using the ARGUS [29] and ANSAT [19] codes is described in Sec. 3. Section 4 describes a generalized theory of rf convection [20,27] and shows that it gives good agreement with the SOL density profiles measured by the reflectometer. A discussion is also given of the 
dependencies of the particle flux to the FS on rf power, phasing and antenna-plasma separation. Section 5 discusses the FS impurity modeling studies and the comparison with the titanium concentration data. A summary and discussion is given in Sec. 6.

\section{TFTR Experimental Arrangement and Results}

The TFTR ICRF system has demonstrated successful high-power coupling into Lmode and supershot plasmas in a variety of heating and current drive regimes with a variety of antenna geometries [30, 31]. Minority heating has been achieved in D-T (H) and D-T ( $\left.{ }^{3} \mathrm{He}\right)$ plasmas and fast wave current drive (FWCD) and mode conversion current drive (MCCD) experiments have been carried out in ${ }^{3} \mathrm{He}-{ }^{4} \mathrm{He}-\mathrm{D}$ plasmas.

The rf sheath analyses described in this paper make use of recent data for $0, \pm \pi / 2$, and $\pi$ phasings, but it is useful to first review some observations from earlier experiments and to comment on their relation to the present work. In its early days, the TFTR ICRF system consisted of two double-strap antennas located in adjacent bays of the tokamak (Bays L and M). Most of the early data on ICRF-edge physics and impurity generation was taken with the original Bay-M antenna configuration (referred to here as the "old Bay-M" antenna). It was found that strong antenna-edge plasma interactions occurred which were dependent on both the antenna phasing and rf power $\mathrm{P}_{\mathrm{rf}}$ [32]. First, a sharp increase in the titanium (Ti) influx from the FS of the powered antenna was observed in 0 phasing for $\mathrm{P}_{\mathrm{rf}}>1 \mathrm{MW}$, whereas a negligible Ti influx was observed for $\pi$ phasing with up to $2.8 \mathrm{MW}$ of power. Also, the heating efficiency was found to be only $50 \%$ in 0 phasing, but $90 \%$ in $\pi$ phasing. It was speculated that the higher impurity level with the in-phase spectrum might have been related to the weaker first-pass absorption [32]. Second, a visible glow was observed in 0 phasing on the bottom of the FS and on certain field lines connecting to the FS $[32,33]$.

The FS protection limiter (also referred to as the "antenna bumper limiter") was later moved forward to intercept many of the field line connections to the Faraday screen. 
The result was a decrease in the observed edge interactions on the modified ("new BayM") antenna and a significant improvement in its power handling abilities [14]. Fig. 1 shows a sketch of the relation of the new Bay-M antenna and the two poloidal limiters (referred to as the "rf limiters") adjacent to Bays $\mathrm{K}$ and $\mathrm{N}$, which protected the antennas from the high density edge plasma. The FS and adjacent bumper limiters are not shown. (A description of the tangency surfaces defined by the complete set of limiters and their typical radial locations is given in Sec. 4.1.) It will be seen that the rf sheath voltage analysis described in Sec. 3 is qualitatively consistent with the improved power handling of the modified Bay-M antenna. In the old-Bay $\mathrm{M}$ configuration, the long field line connections to the rf limiters, together with substantial rf magnetic flux linkage through the slotted antenna sidewalls, produced high-voltage screen-limiter sheaths. In the new Bay-M antenna configuration, the long field line connections were reduced.

The present paper is mainly concerned with data taken with the antenna set used in 1994 - 1995. This TFTR ICRF system consisted of three double-strap antennas in Bays $\mathrm{K}$ - M driven by six generators. Each antenna was center-grounded and end-fed using a resonant transmission line loop. The maximum generator power available to the set of antennas was $12 \mathrm{MW}$. The antennas were driven by pairs of rf generators (one per strap) and could be operated at toroidal phases other than $\pi$. A feedback system was used to maintain constant relative antenna phase. The rf generators were tunable over the range 40-80 MHz, and the antenna matching systems permitted operation at 43 or $63.6 \mathrm{MHz}$. An important feature of this antenna system was the installation of a differential-phase microwave reflectometer on the Bay-K antenna. The reflectometer operated in the 90-118 $\mathrm{GHz}$ range using the extraordinary mode (x-mode) and viewed the plasma through a diagnostic port located in the geometric center of the Bay-K Faraday screen. Each antenna was equipped with titanium-carbide-coated Faraday screens and graphite frame limiters. The screen on the Bay-K antenna had a single row of oval rods slanted $6^{\circ}$ to give approximate alignment with the equilibrium magnetic field. The screens on the Bay-L 
and Bay-M antennas had double rows of circular rods oriented in the toroidal direction. For most ICRF applications, the plasma was partially limited on the rf poloidal limiters in order to maximize the antenna loading.

A number of interesting edge physics experiments were carried out with these antennas to test the predictions of the $\mathrm{rf}$ sheath model. In one experiment [26], the Bay-K reflectometer was used to measure the density profile modifications near the antenna in 0 and $\pi$ phasings for a D-He plasma. Only the Bay-K antenna was powered for these shots and a relatively low power was used $\left(\mathrm{P}_{\mathrm{rf}}<1 \mathrm{MW}\right)$. When the rf power was applied, the density at the antenna was depleted and the density profile was flattened in front of the antenna. The radial extent of the flattened region was larger in 0 than in $\pi$ phasing and increased with $\mathrm{rf}$ power [26]. Both ponderomotive and $\mathrm{rf}$ sheath effects driven by $\mathrm{E}_{\|}$were investigated to explain this behavior [20]. In Sec. 4 we show that a generalized rf convection model is consistent with the density profile data.

Another important measure of the antenna-plasma interaction is the influx of titanium, and the corresponding increase in $\mathrm{Z}_{\mathrm{eff}}$ and radiated power. It has been established that the Ti impurities in TFTR originate from the TiC-coated Faraday screens [32]. Direct spectroscopic measurements of the Ti influx from the screens are not available, but measurements of spectral lines from highly-ionized Ti atoms can be used to deduce the total $\mathrm{Ti}$ concentration in the core plasma. The interpretation of this data is complicated, because there are two channels by which Ti can enter the plasma: a direct influx from the FS and indirect contributions from Ti atoms which were redeposited on other surfaces and subsequently re-sputtered. The mechanisms contributing to rfenhanced influxes in the two channels are different. The degree to which each channel contributes depends on the antenna design and rf parameters, the degree of SOL impurity screening by ionization, the single pass absorption, and on the conditioning of the limiters. In Sec. 5, the Ti impurity data is compared with calculations of the direct influx 
from the antennas for a small database of similar shots in D-He plasmas with $\pi$ and $\pm \pi / 2$ phasings.

\section{TFTR Sheath Analysis}

One of the new features of our sheath analysis on TFTR is the first application of a suite of codes (the ARGUS antenna code [29] and the ANSAT sheath code [19]) to accurately compute the three-dimensional (3D) rf sheath voltage distribution on the antenna including the full antenna geometry, electromagnetic field structure, and equilibrium field line mapping. The ANSAT algorithm and its application to TFTR is described in detail in Ref. 19; here we give an overview of the results necessary for the calculations in later sections.

To motivate this analysis, we first briefly recall the analytic rf sheath model [9] used in previous work, which gives a good representation of an idealized antenna but is not adequate for the TFTR antennas. In this model, only screen-screen field line connections are considered (front face and gap sheaths), the antenna is assumed to be perfectly aligned with the equilibrium magnetic flux surface (so that the field line contact points on the FS are symmetric about the centerline), and the rf magnetic flux driving the sheath is assumed to come only from the current straps. For sheaths on the front face of the FS, this model predicts a large phasing dependence of the sheath voltage arising from flux cancellation among the current straps. The phasing dependence can be simply illustrated for the case of a field line linking the flux of all the current straps of the antenna. In this case, the sheath voltage $\mathrm{V}$ is given by

$$
\begin{array}{r}
\mathrm{V}=\mathrm{V}_{1} \sin \left(\mathrm{N}_{\mathrm{s}} \phi / 2\right) / \sin (\phi / 2), \\
\mathrm{V}_{1}=\mathrm{V}_{\mathrm{a}}\left(\mathrm{L}_{\mathrm{z}} / \mathrm{N}_{\mathrm{s}} \mathrm{L}_{\mathrm{y}}\right) \tan \theta .
\end{array}
$$

Here, $\mathrm{V}_{1}$ denotes the $\mathrm{rf}$ sheath voltage driven by the rf magnetic flux of a single current strap, $\mathrm{V}_{\mathrm{a}}$ is the antenna voltage from top to bottom along the strap, $\mathrm{N}_{\mathrm{S}}$ is the number of 
straps, $\phi$ is the phase difference between straps, $\mathrm{L}_{\mathrm{y}}$ and $\mathrm{L}_{\mathrm{z}} \propto \mathrm{N}_{\mathrm{s}}$ are the poloidal and toroidal dimensions of the antenna, and $\theta$ is the misalignment angle between the magnetic field line and the FS bars.

For $\mathrm{N}_{\mathrm{s}}=2$, Eq. (1) predicts that $\mathrm{V} / \mathrm{V}_{1}=\{2,1.4$, and 0$\}$ for $\phi=\{0, \pi / 2$, and $\pi\}$, respectively. Such a strong phasing difference between $\pi / 2$ and $\pi$ is not consistent with the impurity influxes and other edge modifications observed on TFTR. In this regard, it has been noted [14] that the analytic model neglects the important linkage of flux from the radial feeders through the slotted sidewalls. The ANSAT results discussed below show that there is a significant residual sheath voltage $\{\mathrm{V}\}$ in $\pi$ phasing on the Bay-M antenna, where $\{\mathrm{V}\}$ denotes the average of $\mathrm{V}$ over the FS surface defined in Eq. (2). This voltage is a maximum in the corners of the antenna and is driven by the rf magnetic flux from the radial feeders. In order to include such complications of realistic antennas as the effects of feeder flux, the nonlinear variation of the voltage along the current strap, screen-limiter field connections, antenna misalignment, capacitive coupling (induced charge) effects, etc., a numerical approach is needed.

The starting point of the numerical rf sheath analysis is the ARGUS calculation of the antenna near fields. The antenna geometry is specified on a 3D grid, the electromagnetic boundary conditions are given, and ARGUS computes the resulting 3D rf electromagnetic field pattern [29]. The ARGUS antenna model can include such structural details as the antenna feeders, antenna box, current straps, Faraday screen, and bumper limiters. The present study uses the ARGUS rf fields calculated in a vacuum; the image currents in the antenna box and the rf field distribution are modified somewhat by the presence of plasma in front of the antenna. The effect of plasma on the rf sheath distribution for the TFTR antenna has been studied in Ref. 19.

The ANSAT sheath analysis code [19] acts as a post-processor to the ARGUS field solution. The inputs to the ANSAT code are the ARGUS data files specifying the antenna structure and the three components of the computed rf electric field solution on a 
3D grid. ANSAT also requires an analytic magnetic field mapping to represent the equilibrium magnetic geometry in the vicinity of the antenna. This mapping can be chosen to simulate many geometric effects (field line tilt, antenna-flux surface mismatch, antenna skew, etc.) which occur in real experiments. For the specified magnetic field, ANSAT locates all points of intersection between the field lines and the nearby material surfaces (antenna box, current straps, Faraday screen, antenna limiters, etc.) and computes the rf sheath driving voltage $V=\int d s E_{\|}$between intersection points for all field line segments. A database is constructed of the sheath voltage $\mathrm{V}$, intersection points $\mathbf{x}_{1}$ and $\mathbf{x}_{2}$, field line length $L$, and sheath area $A_{n}$ projected normal to $\mathbf{B}$ for all field lines in the desired volume. In constructing the ANSAT sheath database, the field line tracing is carried out only in the radial region expected to have a significant plasma density. One can compute the averages $\{\mathrm{V}\}$ and $\{\mathrm{L}\}$ and the total area $\mathrm{A}_{\mathrm{n}}$ for the whole database or for any subset of it. For example, this procedure is useful for comparing the contributions of various classes of rf sheaths, as discussed subsequently. Physical quantities such as the FS impurity generation and sheath power dissipation are easily computed from the database.

The complete ARGUS-ANSAT analysis has been carried out for the Bay-M antenna geometry with horizontal FS bars. The structure file includes a detailed model of the antenna box, current straps, and Faraday screen. Versions without the bumper tiles (simulating old Bay-M) and with the bumper tiles (for new Bay-M) have been compared. A variety of ANSAT diagnostics have been used to display and analyze the sheath database for this antenna [19]. For example, Fig. 2 shows a plot of the field line segments and FS contact points on the Bay-M antenna for a subset of the database. The parameters used here are $\theta=3^{\circ}$ (B field-FS misalignment angle), $\mathrm{R}_{\mathrm{c}}=3.6 \mathrm{~m}$ (toroidal radius of curvature) and $\alpha=2.6^{\circ}$, where $\alpha$ is the angle between the toroidal direction and the FS bars at the antenna midplane (see Fig. 3 of Ref. [9]); unless otherwise stated, these parameters are assumed throughout this paper. Four classes of sheaths are illustrated in 
Fig. 2, each characterized by the location of the contact points and the geometry of the rf magnetic flux linkage: gap (G), screen-screen (SS) or front face, screen-limiter short $\left(\mathrm{SL}_{\mathrm{S}}\right)$, and screen-limiter long $\left(\mathrm{SL}_{\mathrm{l}}\right)$.

In Fig. 3, we show filled high voltage contours on the face of the FS for 0 and $\pi$ phasing of the Bay-M antenna without bumper limiters. The units of $\mathrm{V}$ quoted here correspond to a convenient fixed-power normalization used in the codes; the conversion to units of $\mathrm{kV}$ for arbitrary power is discussed later in this section. Figure 3 shows the sheath contribution to the "hot spots" and erosion points on the FS, as the sheath-induced local power dissipation and the impurity influx are proportional to a power of $\mathrm{V}$. The location of the high voltage sheaths computed here correlates well with the location of the observed FS glow [33] on the Bay-M antenna and corresponds to field line connections between the screen and the rf limiter [19].

The strong phasing dependence predicted by the analytic model for the SS sheaths in the center of the FS (driven by the current strap magnetic flux) is clearly seen in Fig. 3. However, note the weaker phasing dependence of the high voltage points in the corners of the FS (driven by the radial current feeds and possibly also by charge buildup at the corners). The existence of significant SL sheath voltages in the corners in $\pi$ phasing is an important result of the ANSAT analysis and is outside the scope of the analytic sheath model in Eq. (1).

Another way of displaying the phasing dependence is shown in Fig. 4. Here, the poloidal variation of the sheath voltage $\mathrm{V}(\mathrm{y})$ is shown on two flux surfaces for 0 and $\pi$ phasings. One of the radial surfaces is located just behind the FS tangency surface (FSTS) and shows the oscillations in V arising from the FS structure (FS bars, gaps and sidewall slots). The oscillations are much smaller on the other surface, which has a radial location well in front of the FS. Figure 4 shows that the poloidal variation of V occurs on two spatial scales, that of the whole antenna and that of the FS periodicity; the spatial structure is driven both by the radial feeders (at the corners) and the current straps (in the 
center). This conclusion is particularly evident from Fig. 4(b). For $\pi$ phasing, V is large near $\mathrm{y}= \pm 0.5 \mathrm{~m}$ because of the feeder flux contribution, but $\mathrm{V} \rightarrow 0$ near $\mathrm{y}=0$ because of the cancellation of the flux from the two current straps. The same peaking of the sheath voltage in the corners of the antenna is evident in 0 phasing, but in this case the current strap contributions give an additional large contribution near $\mathrm{y}=0$. The small scale poloidal variation seen in Fig. 4 is also important, as we shall see in Sec. 4, because it drives rapid $\mathbf{E} \times \mathbf{B}$ convection of particles into the FS which modifies the density profile near the antenna [12, 20, 27]. It should be noted that Fig. 4 employs numerical smoothing, both to compensate for loss of resolution at the finest scales and to simulate the smoothing effect of viscous dissipation on the convection [12], which damps modes on short-scale lengths.

A detailed statistical description of the sheath voltage distribution can be obtained from the database by plotting histograms $\mathrm{N}(\mathrm{V})$ of the number $\mathrm{N}$ of contact points with sheath voltage $\mathrm{V}$, and by making scatter plots of V versus the field line length L [19]. These exercises are valuable for gaining insight into how to divide the sheath database into physically meaningful classes, so that the contribution of each sheath class to the total sputtered influx or dissipated power can be calculated. The average $\{Q\}$. of a quantity Q over the database is defined [19] as

$$
\begin{gathered}
\{\mathrm{Q}\} \equiv \frac{\int \mathbf{d A} \cdot \mathbf{b} Q}{\int \mathbf{d} \mathbf{A} \cdot \mathbf{b}}, \\
=\frac{\sum_{j}\{Q\}_{j}\left(A_{n}\right)_{j}}{\sum_{j}\left(A_{n}\right)_{j}},
\end{gathered}
$$

where the average is weighted by the area of the sheaths projected normal to $\mathbf{b}=\mathbf{B} / \mathbf{B}$, i.e. the flux tube area. If the database is divided into several mutually-exclusive classes of points, and the sum over classes is denoted by the subscript $\mathrm{j}$, it can be shown that Eq.(2a) leads to Eq. (2b), where $\{\mathrm{Q}\}_{\mathrm{j}}$ denotes the area-weighted average and $\left(\mathrm{A}_{\mathrm{n}}\right)_{\mathrm{j}}$ the total flux tube area for the jth class. 


\section{Table 1}

\section{Sheath analysis for the Bay-M antenna}

\section{without bumper limiters and with $\theta=3^{\circ}$ misalignment.}

Shown for each sheath class are the total sheath area $A_{n}$ projected normal to $\mathbf{B}$ (i.e. the flux tube area), the average field line segment length $\{\mathrm{L}\}$, the average distance $\{\Delta \mathrm{r}\}$ of the sheaths behind the FS tangency surface, and the average sheath driving voltage $\{\mathrm{V}\}$ in ANSAT units for $0, \pi / 2$ and $\pi$ phasings. The averages are weighted by the flux tube area. The normalization of the sheath voltages is given in Eq. (3).

\begin{tabular}{|c|cccccc|}
\hline $\begin{array}{c}\text { Sheath } \\
\text { Class }\end{array}$ & $\begin{array}{c}\mathrm{A}_{\mathrm{n}} \\
\left(\mathrm{cm}^{2}\right)\end{array}$ & $\begin{array}{c}\{\mathrm{L}\} \\
(\mathrm{cm})\end{array}$ & $\begin{array}{c}\{\Delta \mathrm{r}\} \\
(\mathrm{cm})\end{array}$ & $\begin{array}{c}\{\mathrm{V}\}_{0} \\
(\mathrm{~A} . \mathrm{U} .)\end{array}$ & $\begin{array}{c}\{\mathrm{V}\}_{\pi / 2} \\
(\mathrm{~A} . \mathrm{U} .)\end{array}$ & $\begin{array}{c}\{\mathrm{V}\}_{\pi} \\
(\mathrm{A} . \mathrm{U} .)\end{array}$ \\
\hline & & & & & & \\
SS & 116 & 14 & 0.4 & 0.15 & 0.14 & 0.10 \\
SL short & 80 & 11 & 0.3 & 0.64 & 0.51 & 0.33 \\
SL long & 3 & 55 & 0.0 & 0.90 & 0.70 & 0.37 \\
& & & & & & \\
\hline
\end{tabular}


Table 1 presents a summary of the sheath class analysis for the Bay-M model antenna with $\theta=3^{\circ}$. For simplicity, the bumper limiters were not included in this calculation. The more complicated sheath geometry in the presence of bumpers is discussed in Ref. 19. The effect of the bumper tiles is to reduce the average field line length $\{\mathrm{L}\}$ and sheath voltage $\{\mathrm{V}\}$. For the Bay-M antenna, the bumpers reduce the antenna-averaged sheath voltage by about $30 \%$ [19]. This is in qualitative agreement with the observed decrease in antenna-plasma interactions with the new Bay-M antenna configuration, as described in Sec. 2.

Table 1 shows that the SL voltages are larger than the SS voltages by a factor of 4 - 6 depending on the phasing. Thus, SL sheaths are expected to dominate local interactions like "hot spots", but the SL contribution to antenna-averaged interactions (such as total impurity sputtering) is limited because the area covered by the SL sheaths is relatively small compared to the SS sheaths. Applying Eq. (2b) to the results of Table 1 yields the following values for the antenna-averaged sheath voltage: $\{\mathrm{V}\}=0.36,0.30$, and 0.20 for $0, \pi / 2$ and $\pi$ phasings, respectively. Thus, the ANSAT calculation yields only about a factor of 2 variation in the sheath voltage for these antenna phasings.

We conclude this section by discussing the questions of interpretation and normalization of the sheath voltage. On TFTR the antennas are grounded in the center and fed at the top and bottom with a relative phase difference of $\pi$, so that an rf voltage difference of amplitude $\mathrm{V}_{\mathrm{a}}$ is generated between the two ends of the current strap. We define the sheath driving voltage $\mathrm{V}$ relative to this maximum antenna voltage $\mathrm{V}_{\mathrm{a}}$. In the language of the simple capacitor-plate sheath model [7], the voltage V is a "peak-to-peak" voltage with a rectification factor of 0.3 , as discussed further in Sec. 4 . The voltage normalization is obtained using the relation $\mathrm{P}_{\mathrm{rf}}=\left(\mathrm{R}_{\mathrm{L}} / 2\right)\left(\mathrm{V}_{\mathrm{a}} / \mathrm{Z}_{\mathrm{o}}\right)^{2}$, where $\mathrm{R}_{\mathrm{L}}$ and $\mathrm{Z}_{\mathrm{o}}$ are the antenna loading resistance and impedance, respectively, and $\mathrm{P}_{\mathrm{rf}}$ is the $\mathrm{rf}$ power delivered to the antenna. For the TFTR Bay-M antenna, a peak-to-peak voltage $\mathrm{V}_{\mathrm{a}}=40$ $\mathrm{kV}$ appears across the current straps at an rf power of about 1.25 MW per antenna for 
typical plasma conditions. Scaling the value of $V_{a}=\int d y E_{y}$ in ANSAT units (A.U.), we obtain the Bay-M normalization [19]

$$
\mathrm{V}(\mathrm{kV})=\mathrm{V} \text { (A.U.) } \mathrm{C}_{\mathrm{N}}\left[\mathrm{P}_{\mathrm{rf}}(\mathrm{MW})\right]^{1 / 2}
$$

with $\mathrm{C}_{\mathrm{N}}=3.1$. This factor depends on $\mathrm{R}_{\mathrm{L}}$ and $\mathrm{Z}_{\mathrm{O}}$, so it will vary slightly among the different antennas and will depend on phasing and plasma position. The value of $\mathrm{C}_{\mathrm{N}}$ used here corresponds to a shot with good antenna loading, and $\mathrm{C}_{\mathrm{N}}$ might be a factor of 2 larger for cases with poor loading. However, for the purpose of this paper, only factor of 2 accuracy is required for $\mathrm{V}$, and we will therefore use this normalization for all phasings and antennas in the calculations of Secs. 4 and 5.

\section{Generalized Convective Cell Model}

One of the most interesting and direct tests of rf sheath theory on TFTR is the measurement of the SOL density profile in front of the antenna using a microwave reflectometer [26]. In this section, we discuss how the reflectometer measurements can be understood in terms of a new model of $\mathbf{E} \times \mathbf{B}$ convection based on rf sheath theory. A brief report of this work was given previously in a conference report [20]. Here, we extend that work and compare the reflectometer data with a recently developed onedimensional (1D) transport model [27] which includes the combined effects of diffusion and convection. This work provides an explanation for the increased radial transport in the SOL (with some evidence for steady-state convection) observed during rf on several tokamaks (see the discussion in Ref. 3 and references therein). After showing that the model gives good agreement with the TFTR reflectometer data, it is then used to compute the radial ion flux to the antenna needed for the impurity calculations in Sec. V. 


\subsection{Motivation}

Rf convective cells (CC) are driven by the $\mathbf{E} \times \mathbf{B}$ drifts resulting from the spatial variation of the rectified $\mathrm{rf}$ sheath potential $\phi(\mathrm{x}, \mathrm{y})[12]$. Here, $\mathrm{x}$ and $\mathrm{y}$ denote the radial and poloidal coordinates in the vicinity of the antenna. The steady-state potential $\phi$ is simply related to the sheath driving voltage $\mathrm{V}$ by $\phi=0.3 \mathrm{~V}+3 \mathrm{~T}_{\mathrm{e}}$, where the second term comes from the Bohm sheath and is typically small [7] . As seen in Fig. 4, $\phi$ has rapid oscillations in y due to the periodic FS structure and $\phi$ decays radially away from the antenna, producing both $\mathrm{E}_{\mathrm{x}}$ and $\mathrm{E}_{\mathrm{y}}$ components of the DC electric field. The flow lines for the $\mathbf{E} \times \mathbf{B}$ drift (contours of constant $\phi$ ) take the form of convective cells in front of the antenna $[12,20,27]$.

The most important consequence of the rf convection is to increase the total plasma flux $\Gamma_{\mathrm{X}}$ to the antenna and thus the strength of the antenna-plasma interaction. The plasma flux is given by

$$
\Gamma_{\mathrm{x}}=-\mathrm{D} \frac{\partial \mathrm{n}}{\partial \mathrm{x}} \pm \mathrm{n} \mathrm{v}_{\mathrm{x}}
$$

where $\mathrm{D}$ is the local diffusion coefficient, $\mathrm{n}$ the local density, and $\mathrm{v}_{\mathrm{X}}$ is the radial component of the CC velocity. Whereas the diffusive flux is typically directed towards the antenna, the sign of the convective term is opposite on each half of the eddy. In the original CC treatment [12], the effect of the convection was modeled by an enhanced diffusion coefficient, $\mathrm{D} \rightarrow \mathrm{D}_{\mathrm{B}}+\mathrm{D}_{\mathrm{rf}}$, where $\mathrm{D}_{\mathrm{B}}$ is the Bohm diffusion coefficient and $\mathrm{D}_{\mathrm{rf}}$ was estimated as $\mathrm{D}_{\mathrm{rf}} \approx \mathrm{v}_{\mathrm{x}} \Delta \mathrm{x}$ with $\Delta \mathrm{x}$ the radial scale length of the eddy. This model has some useful features. It gives an estimate of the convective enhancement of the plasma flux to the antenna required to give good agreement with the JET impurity data [9-11], and it provides a good candidate mechanism to explain the observed dependence of the JET ICRF-induced H-modes on the antenna phasing [12]. However, we will see that this simple model overestimates the magnitude of $\Gamma_{\mathrm{X}}$ and does not accurately predict the behavior of the density at the antenna, because it does not properly take into account the convective nature of the particle flux. 
If the density is regarded as fixed at some reference surface (e.g. the rf limiter surface), the original CC model predicts a (diffusive) density rise at the antenna with rf power. This prediction was directly tested on TFTR by reflectometer measurements [26] of the SOL density profile in front of the Bay-K antenna. The density at the FS was observed to decrease with $\mathrm{rf}$ power, in contradiction to the predictions of the original $\mathrm{CC}$ model. An example of this data is shown in Fig. 5, where the measured density profiles for the ohmic and rf phases are shown for two shots having similar rf power and plasma conditions but different relative phasings of the current straps: 0 phasing (Fig. 5a) and $\pi$ phasing (Fig. 5b). (Depending on the shot, the ohmic profile is measured either just before the turn-on or just after the turn-off of the rf power.) The nearly linear slope of the ohmic profiles $\left(\mathrm{n}^{\prime \prime} \approx 0\right.$, where $\left.{ }^{\prime}=\mathrm{d} / \mathrm{dx}\right)$ indicates the importance of recycling and ionization in fueling the SOL for these shots, which used helium as the majority gas and thus had a recycling coefficient of unity. The most striking features of the rf profiles are the density depletion in front of the antenna and the local flattening of the density profile near the FS. The region of depleted density is larger for 0 than for $\pi$ phasing, and the width of the depleted region increases with $\mathrm{rf}$ power. These trends are consistent with an rf sheath-driven mechanism. A similar density depletion was observed with probes on TEXTOR [28] during screenless operation and was attributed to ponderomotive density expulsion. It is found that the ponderomotive potential is generally small for TFTR parameters, and ponderomotive density expulsion probably cannot account for the data. For example, we estimate that coupling $1 \mathrm{MW}$ on a TFTR antenna gives a typical $\mathrm{E}_{\|}$of $30 \mathrm{~V} / \mathrm{cm}$ just in front of the FS, resulting in a ponderomotive potential of only $5 \mathrm{~V}$. Here we explore the alternative explanation of density pump-out by sheath-induced $\mathbf{E} \times \mathbf{B}$ convection and show that it gives good agreement with the TFTR measurements.

In interpreting Fig. 5 and later figures in this section, it is necessary to know the location of several reference surfaces. The TFTR edge plasma is composed of radial regions having different values of the parallel connection length $L_{\|}$, separated by the 
following tangency surfaces: the $\mathrm{rf}$ limiter at $\mathrm{x}_{\mathrm{RF}}=359.5 \mathrm{~cm}$, the bumper limiter at $\mathrm{x}_{\mathrm{B}}=$ $360.5 \mathrm{~cm}$, and the FS tangency surface at $\mathrm{x}_{\mathrm{FS}}=360.7 \mathrm{~cm}$. The reflectometer is located at the geometric center of the FS, and the front face of the FS at that point is located at $\mathrm{x}_{\mathrm{FF}}=$ $361 \mathrm{~cm}$. Also, $\mathrm{x}_{\mathrm{FF}}$ is the point where the density is assumed to vanish in the convective cell modeling described subsequently. All radial positions $\mathrm{x}$ are defined at the toroidal location corresponding to the center of the antenna $(z=0)$. Here, we define "tangency surface" to mean the flux surface closest to the main plasma which intersects the given object; the first point of intersection is generally not at $\mathrm{z}=0$, so the tangency surface is mapped back to the antenna centerline to obtain the value of the coordinate $\mathrm{x}$. The estimate of the positions of these surfaces takes into account the effect of magnetic field ripple and has an uncertainty of about $2 \mathrm{~mm}$. The numbers given here are the best estimates for the Bay-K SOL during the shots of interest.

The challenge in interpreting this data was to find a mechanism whereby strong rf convection could provide density depletion, rather than enhancement, at the FS. The resolution of this problem [20] was suggested by our ANSAT calculations of the sheath potential structure. Figure 6 shows the computed rf convective flow contours for the Bay$\mathrm{M}$ antenna with 0 phasing and a misalignment angle of $\theta=3^{\circ}$. (We use the Bay-M results because no ARGUS simulations exist for the Bay-K antenna. The antennas are similar enough that the qualitative features of the rf sheath distribution should be the same for both antennas.) The important point to note in Fig. 6 is that there are two distinct radial regions of convection. First, there is a region of closed CCs centered on the bumper limiter tangency surface (BTS), which is the point of maximum sheath voltage in the radial direction. The existence of these cells is easily inferred from analytic sheath models, and they were the focus of the earlier work [12]. The new feature revealed by the ANSAT calculation is the existence of a second region of open $\mathrm{CCs}$, which can sweep particles rapidly into the FS on a timescale of the eddy time, $\tau_{\mathrm{c}} \approx \Delta \mathrm{x} / \mathrm{v}_{\mathrm{x}}$. If the eddy time is shorter than the parallel sonic flow time between the bumper limiters $\left(\tau_{\mathrm{c}}<\tau_{\|}=\mathrm{L}_{\|} / \mathrm{c}_{\mathrm{S}}\right.$, 
where $\mathrm{c}_{\mathrm{S}}$ is the ion sound speed), the rf convection reduces the overall particle confinement time $\tau_{\mathrm{p}}=\tau_{\mathrm{c}} \tau_{\|} /\left(\tau_{\mathrm{c}}+\tau_{\|}\right)$and acts as an enhanced particle sink. This "enhanced pumping" effect was not considered in the original CC model [12]. Thus, the new picture of rf convection [20] based on Fig. 6, which we refer to as the "generalized CC model," has two components: i) the open CCs flatten the density profile behind the bumper limiter and deplete the density near the FS; ii) the closed CCs mix particles originating on both sides of the BTS, thereby smoothing out the abrupt radial jump in $\tau_{\mathrm{p}}$ and also effectively reducing the particle confinement in the region beyond the BTS. All of these effects are qualitatively consistent with the measured density profiles in Fig. 5.

\subsection{The Model}

For a quantitative comparison with the data, the generalized CC model is implemented using a "two sheet" fluid approach developed recently [27]. The competition between convection and diffusion in the radial direction is treated by introducing two "sheets" for the density function, one for each direction of convective flow. Denoting the density on the two sheets by $\mathrm{n}_{ \pm}$, this model solves the density equations

$$
\frac{\partial}{\partial \mathrm{x}}\left(-\mathrm{D} \frac{\partial \mathrm{n}_{ \pm}}{\partial \mathrm{x}} \pm \mathrm{n}_{ \pm} \mathrm{v}\right)+\frac{\mathrm{n}_{ \pm}}{\tau_{\|}}=S \mathrm{n}_{ \pm}
$$

where $v=\left|v_{X}\right|$ is a typical CC velocity and $S=n_{0}\langle\sigma v\rangle$ is the ionization source. The sheets correspond to averages over the regions in $y$ where $v_{X}$ is of one sign, i.e. $n_{+}\left(n_{-}\right)$ corresponds to flow directed towards (away from) the antenna. The total density is given by a sum over sheets, $\mathrm{n}=\mathrm{n}_{+}+\mathrm{n}_{-}$. The $\mathrm{CC}$ model is completed by specifying appropriate boundary conditions (BCs), discussed subsequently, to connect the sheets across radial regions of different flow geometries. One of the novel features of the model is that particles swept into the FS are not allowed to return to the SOL, which is the essential physical point leading to the density depletion. The results of the two-sheet formalism have been compared to a Monte Carlo simulation of the same model problem and good 
agreement was obtained [27]. An important result of these calculations is that the density profile radial scale length $\lambda_{\mathrm{n}}$ becomes very long where the convective and diffusive fluxes add and very short where the two fluxes tend to cancel. The density profiles obtained with this model look qualitatively very similar to the measured TFTR ones and support the idea that convective sweeping of plasma into the FS is responsible for the observed density depletion.

Two versions of the $\mathrm{CC}$ model are employed in this work, which differ in the assumed radial velocity profile $\mathrm{v}(\mathrm{x})$ and in the corresponding $\mathrm{BCs}$. In the "exponential CC model" the radial velocity $\mathrm{v}$ is approximated by

$$
\mathrm{v}(\mathrm{x})=\mathrm{v}_{\mathrm{a}} \exp \left[\left(\mathrm{x}-\mathrm{x}_{\mathrm{FS}}\right) / \mathrm{L}_{\mathrm{rf}}\right], \quad\left(\mathrm{x}_{0}<\mathrm{x}<\mathrm{x}_{4}\right)
$$

where $\mathrm{v}_{\mathrm{a}}$ denotes the magnitude of the radial $\mathbf{E} \times \mathbf{B}$ velocity at the FS tangency surface, and $\mathrm{L}_{\mathrm{rf}}$ is the radial decay length of $\mathrm{v}_{\mathrm{x}}$, assumed to be constant for simplicity. $\mathrm{L}_{\mathrm{rf}}$ is chosen here to be of order the average skin depth $\delta_{\mathrm{e}}=\mathrm{c} / \omega_{\mathrm{pe}}$ in the private SOL of the antenna. The notation $\mathrm{x}_{\mathrm{j}}(\mathrm{j}=0$ to 4$)$ will be defined below. In the "square-well $\mathrm{CC}$ model" the velocity is taken as

$$
\begin{array}{ll}
\mathrm{v}(\mathrm{x})=\mathrm{v}, & \left(\mathrm{x}_{1}<\mathrm{x}<\mathrm{x}_{4}\right) \\
\mathrm{v}(\mathrm{x})=0, & \left(\mathrm{x}_{0}<\mathrm{x}<\mathrm{x}_{1}\right)
\end{array}
$$

and the closed CC width is defined as $\Delta=\mathrm{x}_{3}-\mathrm{x}_{1}$. The algorithm for relating the parameters $(\mathrm{v}, \Delta)$ in the square-well model to the parameters $\left(\mathrm{v}_{\mathrm{a}}, \mathrm{L}_{\mathrm{rf}}\right)$ in the exponential model is explained subsequently in the discussion of Fig. 8. Both models are useful for analyzing the reflectometer data. The exponential CC model gives the greatest accuracy in fitting the experimentally measured density profiles $\mathrm{n}_{\mathrm{e}}(\mathrm{x})$. The square-well model allows analytic solutions in each flow region and is less computationally intensive; thus, it is used here for computing the plasma flux $\Gamma_{\mathrm{X}}$ to the FS for a database of shots. 
The two-sheet CC model is applied to a flow pattern which is an idealization of the flow contours shown in Fig. 6. In the simplified picture (see Fig. 1 of Ref. [27]) there is a region $\left(\mathrm{x}_{0}<\mathrm{x}<\mathrm{x}_{1}\right)$ where the radial transport is dominated by diffusion and only a single function $\mathrm{n}(\mathrm{x})$ is required to specify the density; a region of closed convective cells $\left(\mathrm{x}_{1}<\mathrm{x}<\mathrm{x}_{3}\right)$, with $\mathrm{x}_{2}$ denoting the center of the cells, where both sheets $\mathrm{n}_{+}(\mathrm{x})$ and $\mathrm{n}_{-}(\mathrm{x})$ are required; and a region $\left(\mathrm{x}_{3}<\mathrm{x}<\mathrm{x}_{4}\right)$ of open convection into the FS, where only $\mathrm{n}_{+}(\mathrm{x})$ is required ( $\mathrm{n}_{-}=0$, see below). In terms of the tangency surfaces defined earlier, simulations such as the one shown in Fig. 6 suggest that $\mathrm{x}_{2}=\mathrm{x}_{\mathrm{B}}$ and $\mathrm{x}_{4}=\mathrm{x}_{\mathrm{FF}}$. Note that the former condition implies that the parallel connection length $L_{\|}$changes discontinuously at $\mathrm{x}_{2}$. In carrying out the data analysis, we choose $\mathrm{x}_{0}$ to be the point where the experimentally-observed density depletion begins [see the discussion of Fig. 7]. The location of the boundary $\mathrm{x}_{1}$ is somewhat arbitrary in the exponential CC model and does not affect the solution since the two-sheet model reduces to the standard diffusion equation when $\mathrm{v}=0$; in the square-well $\mathrm{CC}$ model, $\mathrm{x}_{1}$ is chosen to be the surface at which $\tau_{\mathrm{c}} / \tau_{\|}=\mathrm{C}<1$, where $\tau_{\mathrm{c}} \approx \Delta / \mathrm{v}$ is the local eddy time and $\tau_{\|}$is the parallel transit time [see the discussion of Fig. 8].

The boundary conditions for the two models can now be stated. In the exponential CC model, Eq. (5) is integrated numerically in each flow region and matched across the boundaries; the dominant transport in the three regions is by diffusion (2), closed CCs (4) and open CCs (2), where the numbers in parentheses indicate the order of the system of equations solved in each region. (As an aside, we note that the integration is carried out continuously across the boundary at $\mathrm{x}_{2}$ by using a tanh function to give a smooth transition in $\mathrm{L}_{\|}$.) Thus, the exponential model requires eight BCs, two for each sheet in each region. We apply three conditions at $\mathrm{x}_{1}$ and $\mathrm{x}_{3}$ : continuity of the total density $\mathrm{n}$ and radial flux $\Gamma_{\mathrm{X}}$ (summed over both sheets) and equipartition between the sheets $\left(\mathrm{n}_{+}=\mathrm{n}_{-}\right)$. The latter condition simply reflects particle conservation in a closed eddy. The remaining two BCs are $\mathrm{n}\left(\mathrm{x}_{4}\right)=0$ and $\mathrm{n}\left(\mathrm{x}_{0}\right)=\mathrm{n}_{\mathrm{exp} 0}$. The former condition implements our physical 
assumption that particles are lost upon hitting the FS. The latter condition renormalizes the numerically-obtained density profile to the experimental value $\mathrm{n}_{\exp 0}$ at $\mathrm{x}=\mathrm{x}_{0}$. In the square-well CC model, the discontinuity of $\mathrm{L}_{\|}$at $\mathrm{x}=\mathrm{x}_{2}$ means that the region of closed convection must be divided into two regions for the analytic solution and twelve BCs are required. In addition to the eight $\mathrm{BCs}$ already given, we require continuity of $\mathrm{n}_{+}, \mathrm{n}_{-}, \Gamma_{\mathrm{X}+}$, and $\Gamma_{\mathrm{x}}-\operatorname{across} \mathrm{x}=\mathrm{x}_{2}$.

A number of other approximations are made to simplify the model calculations. The diffusion coefficient $\mathrm{D}$ is assumed constant and chosen to have the local Bohm value in the SOL. The parameters determining the ionization profile $S \tau_{\|}(x)$ are chosen by fitting the model two-sheet density profile with $\mathrm{v}=0$ to the measured ohmic profile for the given shot. The ionization profile is then held constant in computing the model density profile during the rf pulse. This assumption is consistent with the observation that the central density changes with rf by less than $10 \%$ for the shots of interest, implying weak changes in the recycling and ionization sources. The assumption of unchanged $S \tau_{\|}$is useful to reduce the number of free parameters and to investigate the extent to which the difference between the ohmic and rf profiles can be explained by enhanced particle losses rather than modified sources.

\subsection{Testing The Model Against TFTR Data}

The CC theory has been tested against a series of experimental shots in D-He plasmas selected to meet two criteria: i) only the Bay-K antenna was powered and no additional neutral beam heating was used; and ii) reflectometer data exists for the rf phase of the discharge. An experiment was carried out during the MCCD studies which meets these criteria; in this experiment, a comparison of 0 and $\pi$ phasing in similar plasma conditions was carried out over a range of rf power.

Figure 7 shows a comparison of the "best fit" model density profiles with measured ohmic and rf density profiles for one shot from this dataset (the same case as in Fig. 5a having 0 phasing and $\left.\mathrm{P}_{\mathrm{rf}}=0.5 \mathrm{MW}\right)$. The theoretical density profile was obtained 
from the exponential CC model by numerical integration of Eq. (5) subject to the BCs described previously with the convective velocity given by Eq. (6). The model parameters $\mathrm{v}_{\mathrm{a}}$ and $\mathrm{L}_{\mathrm{rf}}$ in Fig. 7 are chosen to give the best fit to the measured rf density profile over a restricted interval $\left(\mathrm{x}_{0}, \mathrm{x}_{\mathrm{FF}}\right)$ in which the $\mathrm{CC}$ model is expected to apply. Recall that $\mathrm{x}_{0}$ is the normalization point where the model density profile is set equal to the experimental one, and $\mathrm{x}_{\mathrm{FF}}$ is the point at the FS where the density is assumed to vanish.

It turns out that the integration interval must be chosen with some care to get a good fit. If $\mathrm{x}_{0}$ is chosen far from the antenna, the fit is dominated by the rf-induced density rise near the rf limiter, which is due to recycling and ionization rather than convection. On the other hand, if $x_{0}$ is chosen too close to the FS, one legislates the density depletion through the density $\mathrm{BC}$ at $\mathrm{x}_{0}$. For shots with ohmic data, we have found that a convenient and physically-reasonable choice is to take $\mathrm{x}_{0}$ to be the crossover point where the measured ohmic and rf density profiles have equal density. Thus, for the calculation shown in Fig. 7 we have taken $x_{0}=359.4 \mathrm{~cm}$ (the left edge of the figure); the other end of the integration interval was chosen to be $x_{F F}=361.0 \mathrm{~cm}$, just beyond the right edge of the figure. This corresponds to the density vanishing inside the FS structure where the parallel connection lengths are very short. The fit of the $\mathrm{CC}$ model to the data is not very sensitive to the choice of $\mathrm{x}_{\mathrm{FF}}$, because the (convective) perpendicular particle flux $\Gamma_{\perp}$ near the FS is not very sensitive to the local density value.

Figure 7 shows that the density profiles obtained from the generalized CC model give a good fit to the experimentally-measured profiles for reasonable choices of the parameters $\mathrm{x}_{0}, \mathrm{~L}_{\mathrm{rf}}$, and $\mathrm{v}_{\mathrm{a}}$. The model profile shows the general features of a linear decay near the rf limiter and a flattened profile near the FS. The agreement between the best-fit model profile and the data in Fig. 7 demonstrates that the mechanism of convective pump-out is a good candidate to explain the measured density profiles.

A more stringent test of the self-consistency of the theory is obtained by comparing the best-fit values of $\mathrm{v}_{\mathrm{a}}$ and $\mathrm{L}_{\mathrm{rf}}$ for the model profiles with those predicted by 
the ANSAT rf-sheath calculations. To obtain a first-principles calculation of $\mathrm{v}_{\mathrm{a}}$, the ANSAT code is used to compute the poloidal average of the radial velocity along the FS. Using the voltage normalization in Eq. (3) and taking $\mathrm{B}=3.4 \mathrm{~T}$ at the antenna, the ANSAT result is converted to experimental units as follows

$$
\mathrm{v}_{\mathrm{a}}(\mathrm{cm} / \mathrm{s})=\mathrm{C}_{\mathrm{v}}\left[\mathrm{P}_{\mathrm{rf}}(\mathrm{MW})\right]^{1 / 2},
$$

where $\mathrm{C}_{\mathrm{V}}=2.9 \times 10^{5}(0$ phasing $), 2.0 \times 10^{5}(\pi / 2$ phasing $)$, and $1.8 \times 10^{5}(\pi$ phasing $)$. These values represent lower bound estimates. Some limitations of the algorithm are discussed in Ref. 19. The averaging procedure is subtle and introduces an uncertainty in the phasing dependence of the coefficient $\mathrm{C}_{\mathrm{V}}$. Reducing the amount of numerical smoothing of the computed sheath potential in ANSAT (as described after Fig. 4) and relaxing the assumption of good antenna loading used in Eq. (3) both give larger values for $\mathrm{v}_{\mathrm{a}}$. A reasonable estimate of the upper bound on $\mathrm{v}_{\mathrm{a}}$ is given by multiplying the coefficient in Eq. (7) by a factor of 2 .

For the case shown in Fig. 7, the best-fit parameters were $\mathrm{L}_{\mathrm{rf}}=0.4 \mathrm{~cm}$ and $\mathrm{v}_{\mathrm{a}}=$ $1.9 \times 10^{5}$. This value of $v_{a}$ is essentially equal to the results of the ANSAT prediction for $v_{a}$ obtained from Eq. (7), and the fitted value of $L_{r f}$ agrees reasonably well with the estimate $\mathrm{L}_{\mathrm{rf}}=\mathrm{c} / \omega_{\mathrm{pe}}$. Thus, the best-fit density profile obtained from the exponential $\mathrm{CC}$ model is in satisfactory agreement with both the experimental data and with a firstprinciples sheath calculation of the radial convection.

The exponential $\mathrm{CC}$ model can also be used to compute the rf-enhanced plasma flux to the antenna, which is a key parameter in determining the strength of antennaplasma edge interactions. Defining $\Gamma_{\mathrm{oh}}=\Gamma_{\mathrm{x}}\left(\mathrm{x}_{\mathrm{FS}}\right)$ for the ohmic density profile and $\Gamma_{\mathrm{rf}}=$ $\Gamma_{\mathrm{X}}\left(\mathrm{x}_{\mathrm{FS}}\right)$ for the density profile during the rf pulse, the enhancement factor $\Gamma_{\mathrm{rf}} / \Gamma_{\mathrm{oh}}$ is found to be about 1.7 for the case shown in Fig. 7 . It is significant that $\Gamma_{\mathrm{rf}} / \Gamma_{\mathrm{oh}}>1$ despite the observed density depletion at the antenna. Evidently, the radial convection (which dominates $\Gamma_{\text {rf }}$ ) is so rapid compared to the background diffusion (responsible for $\Gamma_{\mathrm{oh}}$ ) 
that the radial flux increases despite the drop in local density. The result is a flattened profile and an increased interaction with the antenna, which is consistent with the observation of increased impurity production during the rf pulse, as discussed in Sec. 5 . It is not obvious how increased antenna-plasma interaction could be explained within the confines of a ponderomotive density depletion model.

Another quantitative test of the rf convective cell model is its ability to predict the dependencies of the flux enhancement $\Gamma_{\mathrm{rf}} / \Gamma_{\mathrm{oh}}$ on phasing and $\mathrm{rf}$ power inferred from the data. For this comparison, we employ the simpler square-well version of the CC model. This approximation greatly facilitates the numerical work involved in the data analysis and modeling. The approximations underlying the data analysis are the same as used for Fig. 7, except that the fitting parameters $\left(\mathrm{v}_{\mathrm{a}}, \mathrm{L}_{\mathrm{rf}}\right)$ are replaced here by $(\mathrm{v}, \Delta)$. For each shot, the ionization profile is determined by fitting the model with $\mathrm{v}=0$ to the ohmic density profile, and the optimal parameters $(\mathrm{v}, \Delta)$ for the rf case are obtained by a leastsquares fit of the square-well solution to the measured rf density profile. The best-fit profiles for the ohmic and rf cases are used to infer the ratio $\Gamma_{\mathrm{rf}} / \Gamma_{\mathrm{oh}}$ from the data, giving the data points shown in Fig. 8.

The theoretical curves in Fig. 8 are calculated as follows. The parameters $(\mathrm{v}, \Delta)$ in the square-well $\mathrm{CC}$ model are determined from the quantities $\left(\mathrm{v}_{\mathrm{a}}, \mathrm{L}_{\mathrm{rf}}\right)$ inferred from the ANSAT analysis. The correspondence is based on the following arguments. Since the eddy time is dominated by the slowest velocity at the edge of the $\mathrm{CC}$ farthest from the antenna, we take $v=v_{a} \exp \left(-\Delta / L_{r f}\right)$, where $v_{a}$ is given by Eq. (7). The square-well CC width $\Delta$ is determined by the physical requirement that the local eddy time $\tau_{\mathrm{c}} \approx \Delta / \mathrm{v}$ to reach the FS by convection be shorter than the parallel transit time $\tau_{\|}=\mathrm{L}_{\|} / \mathrm{c}_{\mathrm{s}}$ to flow to the limiter, i.e. $\tau_{\mathrm{c}} / \tau_{\|}<1$. Thus, $\Delta$ is computed by setting $\tau_{\mathrm{c}} / \tau_{\|} \equiv\left(\Delta / \mathrm{L}_{\mathrm{rf}}\right) \exp \left(\Delta / \mathrm{L}_{\mathrm{rf}}\right)\left(\mathrm{c}_{\mathrm{s}} / \mathrm{v}_{\mathrm{a}}\right)$ $\left(\mathrm{L}_{\mathrm{rf}} / \mathrm{L} \|\right)=\mathrm{C}$, where the constant $\mathrm{C}<1$ can be regarded as a normalization or profile factor used to correct for the artificiality of the square-well profile. The theory curves shown in Fig. 8 correspond to the choice $\mathrm{C}=0.5$. 
Figure 8 shows the dependence of $\Gamma_{\mathrm{rf}} / \Gamma_{\mathrm{oh}}$ on antenna phasing and $\mathrm{rf}$ power for the database of shots described previously. To reiterate, the data points correspond to the experimentally-inferred values using the best-fit square-well $\mathrm{CC}$ model profiles, and the lines show the theoretical predictions for 0 and $\pi$ phasings with $v_{a}$ given by Eq. (7). The dotted line displays the upper bound on the theoretical prediction for 0 phasing with $\mathrm{v}_{\mathrm{a}}$ increased by a factor of 2, as discussed after Eq. (7). Thus, the difference between the curves labeled " 0 " and " $2 \times 0$ " gives an indication of the theoretical uncertainty. The flux enhancement inferred from the square-well CC model for the shot depicted in Fig. 7 is about 2.2, slightly higher than the value 1.7 inferred from the exponential CC model, which gives an indication of the uncertainty in the modeling of the data points in Fig. 8. The main points to be drawn from Fig. 8 are the following: i) flux enhancement during the $\operatorname{rf}$ phase $\left(\Gamma_{\mathrm{rf}} / \Gamma_{\mathrm{oh}}>1\right)$ is present for all shots; ii) the data points show that the flux enhancement in the two phasings differs by less than a factor of 2 , and the theoretical curves predict a phasing dependence of less than $30 \%$ for $\mathrm{P}_{\mathrm{rf}}<0.8 \mathrm{MW}$; and iii) the theory curves predict a weak dependence on $\mathrm{rf}$ power which is consistent with the data points to within the experimental scatter. We conclude that there is reasonable agreement between the flux enhancement inferred from the measured density profiles and that computed from the ANSAT sheath analysis.

\subsection{Phasing Dependence of the Model}

The CC model may be used to extrapolate to other parameter regimes. For example, the square-well CC model calculations show that the weak dependencies on phasing and rf power observed for the TFTR dataset do not hold for smaller antennaplasma separation. As the antenna is pushed closer to the limiter, the region of density depletion can approach the limiter tangency surface. If the density is assumed to be held constant on this surface by diffusion from the core, and the depletion region can be maintained by sufficiently strong convection, the $\mathrm{CC}$ model predicts a greatly-enhanced 
density gradient and plasma outflux at the limiter tangency surface. Another prediction is that $\Gamma_{\text {rf }}$ becomes much more sensitive to the antenna phasing, because the $\mathrm{CC}$ width and depletion region [see Fig. 5] are phasing-dependent through the rf sheath voltage driving the convection. These points are illustrated in Fig. 9, where we have computed the theoretical curves of $\Gamma_{\mathrm{rf}} / \Gamma_{\mathrm{oh}}$ vs $\mathrm{P}_{\mathrm{rf}}$ for $0, \pi / 2$ and $\pi$ phasings using the same parameters as in Fig. 8 but with the antenna-plasma separation $\Delta \mathrm{x}=\mathrm{x}_{\mathrm{FF}}-\mathrm{x}_{\mathrm{RF}}$ reduced from $12 \mathrm{~mm}$ to 5 $\mathrm{mm}$. For these parameters, the 0 phasing curve shows already an eight-fold enhancement of the plasma flux at $1 \mathrm{MW}$ and rises rapidly at higher power as the $\mathrm{CC}$ width $\Delta$ approaches $\Delta \mathrm{x}$; the other two phasings show only modest flux enhancement $\left(\Gamma_{\mathrm{rf}} / \Gamma_{\mathrm{oh}}<3\right)$ for these parameters. The singularity in $\Gamma_{\mathrm{X}}$ at large power means that the constraint of fixed density at the rf limiter is unphysical, and the density depletion region simply moves beyond the limiter. The JET ICRF H-mode experiment discussed in Ref. 12 is an example of a case where the rf convection is thought to have penetrated beyond the antenna limiters and influenced the edge plasma confinement during operation with 0 phasing. Figure 9 illustrates the importance of using adequately protected antennas for high-power operation in 0 phasing.

To summarize the discussion in Sec. 4, a model of radial transport in the private SOL of the antenna was described which includes the combined effects of diffusion, convection, and particle losses to the antenna. The CC analysis using this model led to a number of useful results. It was shown that particle pump-out by rf convection is a good candidate mechanism to explain the density depletion in front of the ICRF antennas observed on TFTR. The rf convection increases the plasma outflux to the antenna and therefore increases the strength of other rf sheath interactions, as suggested earlier [12]. For TFTR parameters, the plasma flux to the antenna is only weakly dependent on phasing and rf power, but it was shown that this situation changes dramatically as the antenna-plasma separation decreases. 


\section{Titanium Impurity Analysis}

Another useful indication of ICRF-edge plasma interactions on TFTR is the titanium (Ti) concentration in the core plasma, which is measured by monitoring $\mathrm{Ti}$ spectral lines from high ionization states. The titanium atoms originate at the TiC-coated Faraday screens, but some fraction of the Ti atoms migrate around the machine and are redeposited on other surfaces. As on other tokamaks, the core concentration of the FS impurity ion is observed to increase by a large factor during rf heating. It should be emphasized that the resulting contribution of the titanium influx to $\mathrm{Z}_{\text {eff }}$ is negligible during routine operation on TFTR, but the effect is a useful benchmark for comparison with rf sheath theory. In Sec. 5.1, we discuss the dependence of the Ti concentration data on antenna phasing, rf power, SOL density and limiter conditioning. In Sec. 5.2 we investigate theoretically whether the observed influx can be explained by rf sheathenhanced sputtering at the antennas $[5-7,9]$. The antenna impurity model in this section employs the sheath voltage calculations of Sec. 3, the convective cell analysis of Sec. 4, and the density profiles measured by the reflectometer to give a more accurate estimate of the impurity influx.

\subsection{Impurity Data}

In Fig. 10 we show the time history of the Ti $170 \AA ̊$ spectral line intensity for two shots with identical rf power waveforms but different phasings. This spectral line corresponds to a high ionization state and is thus a measure of the central Ti concentration $\mathrm{n}_{\mathrm{Ti}} / \mathrm{n}_{\mathrm{e}}$. During the $\pi$ phasing shot, there is negligible Ti radiation during the ohmic phase $(\mathrm{t}<2.5 \mathrm{sec})$, whereas there is already a significant background Ti radiation before the $\mathrm{rf}$ power is turned on for the $\pi / 2$ phasing shot. The $\pi$ phasing shot (\#82576) occurred early in the experimental run when the limiters were still clean from the overnight conditioning, whereas the $+\pi / 2$ phasing shot (\#82601) took place later in the day. The ohmic data in Fig. 10 suggests that some of the Ti atoms released from the Faraday 
screen migrate to other points in the machine and are subsequently released in later shots. This observation is consistent with the modeling result in Sec. 5.2 that Ti atoms are heavily screened by ionization in the SOL. The time traces during the rf pulse have a large phasing dependence at the same value of rf power.

The shot history of the rf power and of the Ti $170 \AA$ spectral line intensities at three times during the discharge are shown in Fig. 11 for a sequence of shots (\#82570 82610) comparing $\pi$ and $\pm \pi / 2$ phasings. At $t=1 \mathrm{~s}$, the ohmic plasma is small and limited on the inner wall of TFTR; at $\mathrm{t}=2.1 \mathrm{~s}$, the rf has not yet been turned on but the plasma has expanded to its full size; and at $\mathrm{t}=3.3 \mathrm{~s}$, the plasma is full-sized, the rf power is at its maximum value, and the impurity radiation is near its peak. The relative Ti line intensities at these times (denoted $\mathrm{Ti}_{10}, \mathrm{Ti}_{21}$, and $\mathrm{Ti}_{33}$, respectively) are shown in Fig. 11. The scaling factors of the three Ti curves (discussed in the figure caption) give an idea of the increased impurity release during the rf pulse, approximately a factor of 40 . The ohmic $\mathrm{Ti}$ data $\left(\mathrm{Ti}_{10}\right.$ and $\left.\mathrm{Ti}_{21}\right)$ show a gradual increase and eventual saturation in the course of the run, which is similar in overall shape to the Ti data during the rf pulse (Ti33) but is much less sensitive to the instantaneous rf power. The most interesting feature of the data is the phasing dependence of the Ti release during the rf pulse. There is a clear difference between the $\mathrm{Ti}_{33}$ signal in $\pi$ and $\pm \pi / 2$ phasings at the same rf power, and the difference grows in the course of the run.

The general increase in the ohmic and rf Ti line intensities (and the phasing dependence) with shot number suggests that a significant part of the central Ti concentration may be related to impurity accumulation on the limiters and subsequent resputtering from these surfaces. However, this interpretation is hard to confirm with our limited dataset, because the rf power tends to increase on consecutive shots within each phasing group. An alternative interpretation is that the Ti line intensity on a given shot is independent of the shot history (and therefore the limiter conditioning) and is simply proportional to the instantaneous rf power. To test this idea, the rf power dependence of 
the Tiz3 data is shown explicitly in Fig. 12. Again, the limited data set and the tendency for high power shots to occur late in the sequence make it difficult to draw a firm conclusion. There is a clear phasing dependence between phased $( \pm \pi / 2)$ and unphased $(\pi)$ operation which grows with rf power, and there is a slight difference between $\pm \pi / 2$ phasings which may depend in part on the shot history. The phased data also suggests a threshold effect at $4 \mathrm{MW}$, but there are not enough data points to confirm this effect.

For later comparison to the impurity modeling in Sec. 5.2, we have chosen a small but representative series of shots from the MCCD database of ${ }^{3} \mathrm{He}-{ }^{4} \mathrm{He}-\mathrm{D}$ plasmas. The Ti impurity fractions $\mathrm{f}_{\mathrm{Ti}}$ inferred from soft X-ray spectroscopy are shown in Fig. 13 for three series of shots comparing $\pi$ and $\pm \pi / 2$ phasings at different rf powers. The magnitude of the measured Ti concentration is small $\left(f_{\mathrm{Ti}} \leq 10^{-3}\right)$ for all the shots, but of interest here is the phasing dependence of the Ti concentration, which seems to grow with rf power. Each series of shots shown in Fig. 13 has roughly comparable plasmas and similar values of total $\mathrm{rf}$ power $\mathrm{P}_{\mathrm{rf}}$ (summed over all antennas). The first series used ICRF alone (with $\mathrm{P}_{\mathrm{rf}} \sim 2 \mathrm{MW}$ ); the other two series (with $\mathrm{P}_{\mathrm{rf}} \sim 3$ and $4 \mathrm{MW}$ ) used ICRF together with one neutral beam $\left(\mathrm{P}_{\mathrm{NBI}} \approx 2.3 \mathrm{MW}\right)$. Reflectometer measurements of the SOL density profile are available only for the 3 and $4 \mathrm{MW}$ data series, so the modeling in the next section is restricted to these shots. The $4 \mathrm{MW}$ shots are taken from the experimental run shown in Figs. 11 and 12. Because the neutral beam power is approximately constant for the 3 - 4 MW shots in Fig. 13, the presence of the beams should not influence our conclusions regarding the phasing dependence of the impurity influx. 


\subsection{Impurity Modeling}

In this section, we will examine to what extent an rf-sheath-based sputtering model can explain the data of Sec. 5.1 assuming that the main source of Ti atoms is sputtering of the antenna Faraday screens by ions accelerated in the rf sheaths. The possible role of SOL screening of impurities by ionization, transport to the limiters, and subsequent resputtering is also briefly discussed.

Previous studies on other tokamaks have shown that local sputtering of FS atoms is caused by rf sheaths forming on the antenna surface [2-7, 9-11]. Ions accelerated in the rf sheaths strike the FS surface with an energy $\mathrm{E}=\mathrm{Ze} \phi$, where $\mathrm{Z}$ is the charge state of the ions, $\phi=0.3 \mathrm{~V}+3 \mathrm{~T}_{\mathrm{e}}$ is the rectified rf sheath potential discussed in Sec. 4 , and V > 3 $\mathrm{T}_{\mathrm{e}}$ is the sheath driving voltage computed with the ANSAT code in Sec. 3. During highpower rf heating experiments, the ion energy $\mathrm{E}$ can be comparable to the peak in the sputtering yield curve $\mathrm{Y}(\mathrm{E})$ of the FS material, and the rf-induced influx $\Phi_{\mathrm{FS}}$ of atoms from the FS can be substantial [9]. A fraction $\mathrm{S}$ of the this influx will be screened by ionization in the SOL and will be redeposited on other surfaces (e.g. the rf limiters and the carbon-clad inner wall on TFTR). A fraction $\mathrm{T}=1-\mathrm{S}$ of the Ti neutrals will be transmitted across the last closed flux surface into the core plasma. Thus, the central Ti concentration is due to the sum of two influxes: an influx $\Phi_{1}=\mathrm{T} \Phi_{\mathrm{FS}}$ directly from the antennas and another influx $\Phi_{2} \propto(1-\mathrm{T}) \Phi_{\mathrm{FS}}$ from the limiters, due to subsequent resputtering. We can calculate $\Phi_{1}$ using well-established models $[9,19]$ and compare it with estimates of the total influx $\Phi_{\mathrm{Ti}}=\Phi_{1}+\Phi_{2}$ inferred from an analysis of the Ti line intensity data shown in Fig. 11 . The ratio of $\Phi_{1} / \Phi_{T i}$ obtained in this way will be used to assess the adequacy of the antenna sputtering model to explain the Ti concentration data.

The local influx $\Phi_{\mathrm{FS}}\left(\right.$ atoms s$\left.^{-1}\right)$ of Ti atoms from the Faraday screens of all the antennas is given by $[9,19]$

$$
\begin{aligned}
\Phi_{\mathrm{FS}} & =\mathrm{N}_{\mathrm{A}} \mathrm{n}_{\mathrm{i}} \mathrm{c}_{\mathrm{S}} \mathrm{A}_{\mathrm{n}} \mathrm{Y}, \\
& =\mathrm{N}_{\mathrm{A}} \Gamma_{\perp \mathrm{i}} \mathrm{A}_{\perp} \mathrm{Y},
\end{aligned}
$$


where $\mathrm{N}_{\mathrm{A}}$ is the number of antennas (assumed identical for simplicity), $\mathrm{n}_{\mathrm{i}} \mathrm{c}_{\mathrm{s}}$ is the parallel flux density of ions flowing into the FS along the field line, $\mathrm{A}_{\mathrm{n}}$ is the area of each antenna normal to $\mathbf{B}$ covered by the sheaths, $\Gamma_{\perp \mathrm{i}}\left(\right.$ atoms $\left.\mathrm{cm}^{-2} \mathrm{~s}^{-1}\right)$ is the perpendicular (radial) outflux density of ions to the FS including the effects of both diffusion and convection, $\mathrm{A}_{\perp}=\mathrm{L}_{\mathrm{y}} \mathrm{L}_{\mathrm{z}}$ is the antenna area normal to the plasma outflux, and $\mathrm{Y}=\mathrm{Y}(\mathrm{E})$ is the sputtering yield per incident ion [34]. Equation (8) contains an implicit summation over all species of incident plasma ions and over all relevant classes of rf sheaths (as discussed in Sec. 3). The two forms of Eq. (8) are equivalent by particle conservation across the FS tangency surface $\left(\Gamma_{\|} A_{n}=\Gamma_{\perp} A_{\perp}\right)$. The form in Eq. (8a) is useful for analyzing the separate contributions of the sheath classes [19], whereas the form in Eq. (8b) is more appropriate here, because it allows us to easily compute the average impurity influx from the FS for comparison with the experimental impurity data.

A number of approximations are used in Eq. (8b) for the analysis of each shot: (i) sputtering - We retain only the sputtering due to the majority ion species. Ti selfsputtering is neglected because the ionization mean-free path is long near the antenna and the local density of $\mathrm{Ti}$ ions is expected to be small near the FS under normal conditions. The sputtering yield is taken as $\mathrm{Y}(\mathrm{E}) \approx \mathrm{R}_{\mathrm{y}} \mathrm{Y}_{90}(\mathrm{E})$, where $\mathrm{Y}_{90}(\mathrm{E})$ is the sputtering yield for an ion of energy $\mathrm{E}$ at normal incidence given by Bohdansky [34], and the small angle of incidence enhancement factor $\mathrm{R}_{\mathrm{y}} \approx 1.5$ is based on typical ion orbits [9]. (ii) plasma flux - We estimate the ion flux to the antenna as $\Gamma_{\perp \mathrm{i}} \approx \Gamma_{\perp \mathrm{e}} / \mathrm{Z}$, where the electron outflux $\Gamma_{\perp \mathrm{e}}$ is inferred by fitting the square-well CC model of Sec. 4 to the electron density profiles measured by the reflectometer. The flux measured at the reflectometer position is assumed to be typical of the FS-averaged flux, and the flux measured on Bay-K is assumed to be typical of the other antennas as well.

(iii) sheath voltages - The ion energy $\mathrm{E}$ and sputtering yield $\mathrm{Y}(\mathrm{E})$ are estimated using the sheath voltages computed numerically in Sec. 3. We assume that the Bay-M sheath analysis in Table 1 and voltage normalization in Eq. (3) are approximately valid for all 
antennas. The area-weighted average of the sheath voltage $\{\mathrm{V}\}$ over the antenna surface [see Eq. (2)] is used in computing the projectile energy E. This approximation is valid if the sputtering yield $\mathrm{Y}(\mathrm{E})$ is a weak function of $\mathrm{E}$ in the range of interest. The curves $\mathrm{Y}(\mathrm{E})$ for light ions impacting Ti tend to be nearly flat for the relevant range of energies and to decrease slightly for $\mathrm{E}>1 \mathrm{keV}$. Moreover, the highest voltage sheaths cover a small surface area [see Table 1]. Thus, the highest voltage sheaths are not expected to give a large contribution to the sputtering, and the averaging procedure in Eq. (2) should be adequate.

These approximations should be sufficiently accurate for the present goal of identifying the important parametric trends (e.g. the phasing dependence) of the impurity flux $\Phi_{\mathrm{FS}}$.

The impurity screening can be estimated using the density profiles $\mathrm{n}_{\mathrm{e}}(\mathrm{x})$ measured by the reflectometer. The impurity transmission coefficient $\mathrm{T}$ is defined by

$$
\mathrm{T}=\exp \left[-\mu \int \mathrm{dx} \mathrm{n}_{\mathrm{e}}(\mathrm{x})\right],
$$

where $\mu=\langle\sigma v\rangle / v_{0}$ is the ratio of the ionization rate $\langle\sigma v\rangle \approx 10^{-7}$ to the neutral impurity velocity $\mathrm{v}_{\mathrm{o}}=\left(\mathrm{T}_{\mathrm{n}} / \mathrm{m}_{\mathrm{n}}\right)^{1 / 2}$ (assumed to be in the $\mathrm{x}$ direction normal to the flux surfaces), the neutral temperature $T_{n}$ is estimated in terms of the lattice binding energy $E_{b}$ of the FS atom as $\mathrm{T}_{\mathrm{n}} \approx \mathrm{E}_{\mathrm{b}} / 2$, and the limits of integration extend from the FS tangency surface at $\mathrm{x}_{\mathrm{FS}}=360.7 \mathrm{~cm}$ to the last closed flux surface position defined by limiting the plasma on the inner wall, assumed to be at $x_{L F S}=358.7 \mathrm{~cm}$, about $8 \mathrm{~mm}$ outside the rf limiter. It is assumed here that the temperature $\mathrm{T}_{\mathrm{es}}$ in the $\mathrm{SOL}\left(\mathrm{x}_{\mathrm{LFS}}<\mathrm{x}<\mathrm{x}_{\mathrm{FS}}\right)$, and hence the ionization rate $\langle\sigma v\rangle$, is constant with power. The argument goes as follows. We assume that most of the power delivered to the plasma, $\mathrm{P}_{\text {tot }}$, transports radially across the last closed flux surface and is lost by parallel flow to the limiters; thus, the SOL energy balance condition is $\mathrm{P}_{\text {tot }} \approx \mathrm{P}_{\|}$, SOL $\propto \mathrm{n}_{\mathrm{es}} \mathrm{c}_{\mathrm{s}} \mathrm{T}_{\mathrm{es}} \propto \mathrm{n}_{\mathrm{es}} \mathrm{T}_{\mathrm{es}}{ }^{3 / 2}$, where $\mathrm{n}_{\mathrm{es}}$ is the SOL density, 
implying that $\mathrm{T}_{\mathrm{es}} \propto\left(\mathrm{P}_{\mathrm{tot}} / \mathrm{n}_{\mathrm{es}}\right)^{2 / 3}$. The empirical scaling $\mathrm{n}_{\mathrm{es}} \propto \mathrm{P}_{\text {tot }}$ is found in these experiments, so that it is reasonable to take $\mathrm{T}_{\mathrm{es}} \approx$ constant in this analysis.

The direct influx $\Phi_{1}$ of Ti atoms from the FS to the core plasma, the secondary influx $\Phi_{2}$ from the limiters, and the total influx $\Phi_{\mathrm{Ti}}$ are obtained as follows

$$
\begin{gathered}
\Phi_{1}=\mathrm{T} \Phi_{\mathrm{FS}}, \\
\Phi_{2}=\Phi_{\mathrm{Ti}}-\Phi_{1}, \\
\Phi_{\mathrm{Ti}}=\mathrm{f}_{\mathrm{Ti}} \frac{\left\langle\mathrm{n}_{\mathrm{e}}\right\rangle \mathrm{V}_{\mathrm{p}}}{\tau_{\mathrm{p}}},
\end{gathered}
$$

where $\mathrm{f}_{\mathrm{Ti}}=\mathrm{n}_{\mathrm{Ti}} / \mathrm{n}_{\mathrm{e}}$ is the experimental Ti fraction inferred from soft X-ray spectroscopy using the 169 and $170 \mathrm{~A}^{\circ} \mathrm{Ti}$ spectral line intensities, $\left\langle\mathrm{n}_{\mathrm{e}}\right\rangle$ is the volume-averaged density (approximated here by the line-averaged density, which differs by less than $20 \%$ for these shots $), \mathrm{V}_{\mathrm{p}}=(2 \pi \mathrm{R})\left(\pi \mathrm{a}^{2}\right)$ is the plasma volume, and $\tau_{\mathrm{p}} \approx 0.25 \mathrm{sec}$ is the measured impurity confinement time on TFTR. Thus, $\Phi_{1}$ is calculated from the FS impurity model described above, whereas $\Phi_{\mathrm{Ti}}$ is inferred from the data. Our goal here is to use a combination of theory and measurements to determine the relative sizes of $\Phi_{1}$ and $\Phi_{2}$ and to compare the theoretically-determined phasing dependence of $\Phi_{1}$ with the experimentally-inferred phasing dependence of $\Phi_{\mathrm{Ti}}$.

The modeling results for the Ti influx from the FS of the powered antennas, $\Phi_{\mathrm{FS}}$, vs total rf power is shown in Fig. 14(a), and the dependence of the transmission coefficient $\mathrm{T}$ on $\mathrm{P}_{\mathrm{rf}}$ (through the rf-induced rise in edge plasma density) is shown in Fig. 14(b). The reflectometer data is used to compute both the particle flux $\Gamma_{\perp}$ in Eq. (8b) and the transmission coefficient $\mathrm{T}$ in Eq. (9), thereby reducing the uncertainty in the theoretical modeling. The calculation of $\Phi_{\mathrm{FS}}$ was carried out for the two limiting cases of minimal $\mathrm{C}$ impurities (a pure He plasma with charge state $\mathrm{Z}=1$ ) and maximal $\mathrm{C}$ impurities (a pure $\mathrm{C}$ plasma with charge state $\mathrm{Z}=3$ ) to investigate the role of the plasma composition. The computed Ti influxes were nearly identical in the two cases. Increasing 
$\mathrm{Z}$ to the fully ionized state for either $\mathrm{C}$ or He leads to a decrease in the impurity influx by about a factor of 1.7 when the perpendicular electron flux $\Gamma_{\perp \mathrm{e}}$ is held fixed at the value inferred from the reflectometer data. The reason is that the slightly increased sputtering energy and yield per ion is outweighed by the decreased flux of ions to the screen $\left(\Gamma_{\perp \mathrm{i}} \approx\right.$ $\Gamma_{\perp \mathrm{e}}(\mathrm{Z})$ as $\mathrm{Z}$ is increased. The results shown here are for the He plasma with $\mathrm{Z}=1$.

The main points to note in Fig. 14(a) are that the impurity influx from the FS increases with rf power and that its phasing dependence is weak (less than a factor of 2). The latter point is explained by the fact that neither the antenna sheath voltages (Sec. 3) nor the plasma flux to the antenna (Sec. 4) have strong phasing dependencies for TFTR parameters. Additionally, the sputtering yield is not a strong function of the particle energy, which further reduces the phasing dependence. The impurity transmission coefficient $T$ in Fig. 14(b) is a sensitive function of the SOL density $n_{e s}$ through the ionization cross-section. The values computed here use the measured SOL density profile and therefore automatically take into account the nearly linear increase of $n_{\mathrm{es}}$ with $\mathrm{P}_{\mathrm{rf}}$. For TFTR parameters, the argument of the exponential in Eq. (9) is typically large, so that $\mathrm{T}$ is small and sensitive to small changes of parameters. Titanium is heavily screened $(\mathrm{T}<0.1)$ for these shots and the screening strongly increases with rf power. This result is consistent with the experimental results in Figs. 10 and 11 suggesting measurable Ti redeposition on the material surfaces bounding the plasma.

The last step in the analysis is to estimate the Ti concentration in the core due to the direct (unscreened) influx of Ti atoms sputtered from the FS. A theoretical estimate can be calculated from Eq. (10), $\mathrm{f}_{\mathrm{Ti}}($ theory $)=\mathrm{T} \Phi_{\mathrm{FS}} \tau_{\mathrm{p}} /\left(\left\langle\mathrm{n}_{\mathrm{e}}\right\rangle \mathrm{V}_{\mathrm{p}}\right)$. A plot of $\mathrm{f}_{\mathrm{Ti}}$ (theory) versus $\mathrm{P}_{\mathrm{rf}}$ is similar to the behavior of T shown in Fig. 14(b) and is therefore not shown. Instead, in Fig. 15 we plot the experimentally-inferred total Ti concentration $\mathrm{f}_{\mathrm{Ti}}(\exp$. versus $\mathrm{f}_{\mathrm{Ti}}$ (theory) with arrows indicating the time sequence of the shots. Figure 15 shows that the modeling of direct impurity release from the antenna into the core plasma is in reasonable agreement with the measured Ti concentration for the $\pi$-phasing shots, but 
there is some deviation between the theoretical prediction and the observed $\mathrm{Ti}$ concentration for $\pm \pi / 2$ phasing in the $4 \mathrm{MW}$ shots. This discrepancy is discussed further in the next section.

\section{Summary and Discussion}

The present paper describes an experimental study of some of the important ICRF-edge interactions on TFTR and a comparison with the predictions of rf sheath theory. In the course of this work, the theoretical model was extended by (i) developing a code for quantitative calculation of the antenna sheath potential distribution, and (ii) developing a generalized model for rf-induced convection, including the competition between convection and diffusion and the effect of the antenna as a particle sink. Quantitative comparisons of the unified rf sheath model (including calculations of the antenna voltage, rf-induced convection, rf-enhanced sputtering of the FS, and impurity screening by ionization in the SOL) showed good agreement with experimental measurements of the SOL density modification near the antenna [Sec. 4] and with the FSimpurity generation data in $\pi$ phasing [Sec. 5]. The factor of two agreement obtained in these comparisons is a significant validation of the theory because of the number of experimental constraints imposed on the modeling. In this section, we discuss the significance of these results to the optimization of rf heating and current drive and point out areas for further work.

The role of rf convection [Sec. 4] is central to quantitative modeling of antennaplasma interactions. The observed decrease in the plasma density near the antenna, often accompanied by increased edge and core density, is ubiquitous in ICRF and IBW experiments. An important and unresolved question is the relative importance of $\mathrm{rf}$ convection and ponderomotive density expulsion in explaining the density decrease. For example, it has been suggested that the observed density depression at the antenna during ICRF heating on TEXTOR with screenless antennas is caused by ponderomotive 
expulsion [28]. If this is the case, one expects a decreased flux of plasma to the antenna, thereby minimizing deleterious edge interactions and facilitating screenless operation. In contrast to that work, our estimates for ICRF heating on TFTR using screened antennas show that ponderomotive expulsion is not large enough to account for the data, whereas the quantitative calculations in Sec. 4 show that the convective model gives good agreement with the measured density profiles. If rf convection is the dominant mechanism, the density depression is accompanied by an increased flux to the antenna (Figs. 8 and 9) and increased antenna-plasma interactions. In this regard, it is interesting to recall that an increased DC radial flux was measured by SOL probes during ICRF heating in a different series of experiments on TEXTOR, presumably using screened antennas [24].

In cases where $\mathrm{rf}$ convection dominates background diffusion and ponderomotive effects, the plasma flux to the antenna will increase with rf power despite the fact that the local density drops. This effect can introduce a strong phasing dependence [Fig. 9] and must be taken into account in estimating local antenna-plasma interactions such as the impurity influx and the sheath power dissipation. The increased plasma flux to the antenna can be reduced by using close-fitting antenna protection limiters that protrude several CC eddy widths into the plasma. If the SOL density diffusively decays before encountering the $\mathrm{CCs}$, the flux to the antenna will be minimized. The limiters also serve another useful purpose for antennas with slotted-sidewalls. By intercepting the longest field line connections to the antenna, the limiters can reduce the screen-limiter sheath voltages. As discussed in Sec. 2, the operation of the TFTR Bay-M antenna was substantially improved by increasing the radial protrusion of the bumper limiters.

The FS impurity analysis described in this paper successfully reproduced the observed level of Ti impurities in the central plasma in an order-of-magnitude sense, but it was unsuccessful in explaining all aspects of the data. This raises some questions for further work. One issue is the origin of the rf voltage or power threshold for deleterious 
antenna effects (impurities, arcing) sometimes observed in the experiment. A second, and related, question concerns the origin of the phasing dependence of the Ti concentration for the high power shots analyzed in Sec. 5.1. Given the assumptions used here, the modeling in Sec. 5.2 predicts that neither the direct impurity influx from the antennas, nor the amount of screening of impurities by ionization in the SOL, is strongly phasing dependent.

One possibility for explaining the phasing dependence of the impurity data is that some sheath-related physical effect with a sharp voltage threshold was neglected in the model. Such an effect could produce a large phasing dependence at high power. As just discussed, an example of this behavior is the plasma flux in the limit of 0-phasing and small antenna-plasma separation, as shown in Fig. 9. Another possible source of a voltage threshold is self-sputtering, which could lead to a sharp increase (avalanche) in the sputtered FS atoms when the product of the sputtering yield and the local ionization rate crosses a threshold [9]. Our best estimates using the antenna surface-averaged model of Sec. 5 suggest that these effects (plasma flux, self-sputtering) are not near the threshold voltage for typical TFTR parameters. However, they could be important on localized "hot" spots on the antenna where exceptional conditions prevail. Including some additional physical effect of this type into the present near-field sheath analysis could conceivably bring the FS sputtering analysis into agreement with the impurity data without invoking other impurity sources in the machine.

A second possibility is that far-field sheath effects play an important role in generating impurities. For example, it is possible that the secondary influx of Ti resputtered from the plasma limiters (not computed here) is the main contributor in high power operation with $\pm \pi / 2$ phasings. In this picture, the screening of impurities by ionization in the SOL leads to significant redeposition of $\mathrm{Ti}$ on the limiters, and this $\mathrm{Ti}$ is resputtered by an $\mathrm{rf}$ process which is proportional to $\mathrm{rf}$ power and is more phasingdependent than the local sputtering process at the FS. A candidate mechanism to explain 
the $\mathrm{rf}$ dependence of the limiter sputtering is ion acceleration in far-field sheaths $[8,13$, 35], in which the phasing dependence arises from the $\mathrm{k} \|$ dependence of the fast wave cutoff [36] or from the presence of rf surface or coaxial modes. Neither the available data nor the present state of the modeling allows us to decide whether this type of mechanism is relevant to TFTR.

In conclusion, substantial progress has been made in recent years in understanding and modeling rf sheath processes, and a unified picture is beginning to emerge. This picture can explain many of the observed ICRF-edge interactions on TFTR. More work is needed to fully understand the ICRF-edge interactions observed during low-k $\|$ heating and current drive.

\section{ACKNOWLEDGEMENTS}

The authors wish to thank Y. L. Ho for providing the ARGUS code results used to model the TFTR Bay-M antenna. We also thank the referees for their careful reading of the manuscript and for helpful suggestions on improving the final version of the paper.

This work was supported by the U.S. Department of Energy under Contract Nos. DE-FG03-93-ER54212, DE-FG02-97ER54392, DE-AC02-76-CH03073, and DE-AC0584-OR21400; however, this support does not constitute an endorsement by DOE of the views expressed herein. 


\section{REFERENCES}

[1] see, for example, the Proceedings of the IAEA Technical Committee Meeting on ICRH/Edge Physics, Garching, Germany (North-Holland, Amsterdam, 1990), published in Fusion Engineering and Design, 12 (1990).

[2] J.-M. NOTERDAEME, in Proceedings of the Ninth APS Topical Conference on Applications of Radio-Frequency Power to Plasmas, AIP Conf. Proc. 244, Charleston, SC, 1991 (American Institute of Physics, Woodbury, NY, 1992), p 71.

[ 3] J.-M. NOTERDAEME and G. VAN OOST, Plasma Phys. Controlled Fusion 35 (1993) 1481 .

[4] F.W. PERKINS, Nucl. Fusion 29 (1989) 583.

[5] R. CHODURA and J. NEUHAUSER, in Proceedings of the 16th European Conference on Controlled Fusion and Plasma Heating, Venice (European Physical Society, Petit-Lancy, Switzerland, 1989) Vol. 13B, Part III, p. 1089.

[6] R. VAN NIEWENHOVE and G. VAN OOST, J. Nucl. Mater. 162 - 164 (1989) 288 - 291; R. VAN NIEWENHOVE, "Influence of ICRF on the edge plasma of tokamaks," Doctoral Thesis, Antwerp (1989).

[7] J. R. MYRA, D. A. D'IPPOLITO and M. J. GERVER, Nucl. Fusion 30 (1990) 845; D. A. D'IPPOLITO, J. R. MYRA, M. BURES, M. STAMP and J. JACQUINOT, Fusion Eng. Design 12 (1990) 209.

[8] M. BRAMBILLA, R. CHODURA, J. HOFFMANN, J. NEUHAUSER, J.-M. NOTERDAEME, et al., in Plasma Physics and Controlled Research 1990 (International Atomic Energy Agency, Vienna, 1991), Vol. I, p. 723.

[9] D. A. D'IPPOLITO, J. R. MYRA, M. BURES, and J. JACQUINOT, Plasma Phys. Controlled Fusion 33 (1991) 607.

[10] M. BURES, J. JACQUINOT, K. LAWSON, M. STAMP, H. P. SUMMERS, et al. , Plasma Phys. Controlled Fusion 33 (1991) 937. 
[11] M. BURES, J. JACQUINOT, M. STAMP, D. SUMMERS, D.F.H. START, et al., Nucl. Fusion 32 (1992) 1139.

[12] D. A. D'IPPOLITO, J. R. MYRA, M. BURES, and J. JACQUINOT, Phys. Fluids B 5 (1993) 3603.

[13] J. R. MYRA, D. A. D'IPPOLITO, and M. BURES, Phys. Plasmas 1 (1994) 2890.

[14] R. MAJESKI, P. PROBERT, T. TANAKA, D. DIEBOLD, R. BREUN, et al., in Proceedings of the Ninth APS Topical Conference on Applications of RadioFrequency Power to Plasmas, AIP Conf. Proc. 244, Charleston, SC, 1991 (American Institute of Physics, Woodbury, NY, 1992), p 322.

[15] R. VAN NIEUWENHOVE and G. VAN OOST, Plasma Phys. Controlled Fusion 34 (1992) 525.

[16] M. D. CARTER, D. B. BATCHELOR, and E. F. JAEGER, Phys. Fluids B 4 (1992) 1081.

[17] S. RIYOPOULOS, W. GROSSMANN, A. DROBOT, and M. KRESS, Phys. Fluids B 4 (1992) 3261.

[18] R. MAJESKI, D. A. D'IPPOLITO, Y.L. HO, J. HOSEA, et al., in Proceedings of the 20th European Physical Society Conference on Controlled Fusion and Plasma Physics, Lisbon, (European Physical Society, 1993), Vol. 17C, Part III, p. 977.

[19] J. R. MYRA, D. A. D'IPPOLITO, and Y. L. HO, Fusion Engineering and Design 31, 291 (1996); J. R. MYRA, D. A. D'IPPOLITO, and Y. L. HO, AIP Conf. Proc. 355, 405 (1996).

[20] D. A. D'IPPOLITO, J. R. MYRA, A. C. ENGLAND, G. R. HANSON, J. B. WILGEN, et al., Proceedings of the Eleventh Topical Conference on Radio Frequency Power in Plasmas, AIP Conf. Proc. 355, Palm Springs, CA, 1995 (American Institute of Physics, Woodbury, NY, 1996), p. 467.

[21] D. A. D'IPPOLITO and J. R. MYRA, Phys. Plasmas 3 (1996) 420. 
[22] C. E. THOMAS, Jr., J. H. HARRIS, G. R. HASTE, C. C. KLEPPER, J. T. HOGAN, et al., Fusion Technology 30 (1996) 1.

[23] R. A. MOYER, R. VAN NIEUWENHOVE, G. VAN OOST, D. BORA, R. W. CONN, et al., J. Nucl. Mater. 176 \& 177 (1990) 293.

[24] D. BORA, R. S. IVANOV, G. VAN OOST, and U. SAMM, Nucl. Fusion 31 (1991) 2383.

[25] P. E. MOROZ, J. R. MYRA, and D. A. D'IPPOLITO, Proceedings of the Twelfth Topical Conference on Radio Frequency Power in Plasmas, AIP Conf. Proc. 403, Savannah, GA, 1997 (American Institute of Physics, Woodbury, NY, 1998) p. 467.

[26] G. R. HANSON, A. C. ENGLAND, J. B. WILGEN, F. W. BAITY, D. B. BATCHELOR, et al., Proceedings of the Eleventh Topical Conference on Radio Frequency Power in Plasmas, AIP Conf. Proc. 355, Palm Springs, CA, 1995 (American Institute of Physics, Woodbury, NY, 1996), p. 463.

[27] J. R. MYRA and D. A. D'IPPOLITO, Phys. Plasmas 3 (1996) 699.

[28] R. VAN NIEUWENHOVE, R. KOCH, and G. VAN OOST, Proceedings of the 21 st European Physical Society Conference on Controlled Fusion and Plasma Physics, Montpellier, (European Physical Society, 1994), Vol. 18B, Part II, p. 976.

[29] Y. L. HO, W. GROSSMANN, A. DROBOT, M. D. CARTER, P. M. RYAN, et al., Proceedings of the Tenth Topical Conference on Radio Frequency Power in Plasmas, AIP Conf. Proc. 289, Boston, MA, 1993 (American Institute of Physics, Woodbury, NY, 1994 ) p. 359.

[30] J. R. WILSON, S. BATHA, D. DARROW, J. C. HOSEA, E. JAEGER , et al., Proceedings of the Eleventh Topical Conference on Radio Frequency Power in Plasmas, AIP Conf. Proc. 355, Palm Springs, CA, 1995 (American Institute of Physics, Woodbury, NY, 1996), p. 3. 
[31] R. MAJESKI, J. H. ROGERS, S. H. BATHA, A. BERS, R. BUDNY, et al., Phys. Plasmas 3 (1996) 2006.

[32] J. E. STEVENS, C. BUSH, P. L. COLESTOCK, G. J. GREENE, K. W. HILL, et al., Plasma Phys. Controlled Fusion 32 (1990) 189.

[33] P. COLESTOCK, A. CAVALLO, W. DORLAND, J. HOSEA, G. GREENE, et al., in Proceedings of the Eighth Topical Conference on Radio Frequency Power in Plasmas, AIP Conf. Proc. 190, Palm Springs, CA, 1988 (American Institute of Physics, Woodbury, NY, 1989), p. 189.

[34] J. BOHDANSKY, in Data Compendium for Plasma-Surface Interactions, Nucl. Fusion Special Issue (1984), p. 64; corrected in Nucl. Fusion 24 (1984) 1683.

[35] F. W. PERKINS, "ICRF Impurity Generation and H-mode," Bull. Am. Phys. Soc. 34 (1989) 2093, paper 6S6.

[36] R. MAJESKI, C. K. PHILLIPS, and J. R. WILSON, Phys. Rev. Lett. 73 (1994) 2204. 


\section{FIGURE CAPTIONS}

Fig. 1 Sketch of the TFTR antenna-limiter geometry including the current straps, Faraday screen and rf limiters. Not shown for clarity are the FS bars and the antenna bumper limiters. Also shown are the field line connections giving rise to screen-screen (SS), screen-limiter (SL), and limiter-limiter (LL) sheaths.

Fig. 2 View of a portion of the Bay-M Faraday screen model used in the ARGUSANSAT analysis. Also shown are typical field line connections, contact points; and the sheath classification discussed in the text. [From Ref. 19, Copyright 1996 - Elsevier Science S. A.]

Fig. 3 Filled voltage contours ( $\mathrm{V}>0.4$ in code voltage units) from the ANSAT plasmafacing analysis for the Bay-M antenna with (a) 0 phasing and (b) $\pi$ phasing. [From Ref. 19, Copyright 1996 - Elsevier Science S. A.]

Fig. 4 Poloidal dependence of the sheath potential, V(y), for the Bay-M antenna on field lines at two radial positions (just inside and just outside the FS tangency surface) with (a) 0 phasing and (b) $\pi$ phasing. [From Ref. 19, Copyright 1996 - Elsevier Science S. A.]

Fig. 5 Measured ohmic (dotted line) and rf (solid line) density profiles for (a) shot 84102 (0 phasing, $0.5 \mathrm{MW}$ ICRF power) and (b) shot 84105 ( $\pi$ phasing, $0.55 \mathrm{MW}$ ICRF power). The vertical lines indicate the radial positions of the surfaces tangent to the rf limiter $\left(\mathrm{x}_{\mathrm{RF}}\right)$ and the $\mathrm{FS}\left(\mathrm{x}_{\mathrm{FS}}\right)$.

Fig. $6 \mathrm{CC}$ flow contours computed by ANSAT for the Bay-M antenna with 0 phasing. The radial positions of the bumper limiter $\left(\mathrm{x}_{\mathrm{B}}\right)$ and FS tangency surface $\left(\mathrm{x}_{\mathrm{FS}}\right)$ are shown. Note the two radial regions of convection (across the SOL and into the FS).

Fig. 7 Best fit of the exponential CC model (dashed lines) given by Eqs. (5) and (6) to the measured density profile (solid lines) during the ohmic phase and rf pulse for shot 84102 (0 phasing, 0.5 MW ICRF power). The model density profile is 
matched to the experimental one at $\mathrm{x}_{0}=359.4 \mathrm{~cm}$ (the left edge of the figure) and is assumed to vanish at $\mathrm{x}_{\mathrm{FF}}=361.0 \mathrm{~cm}$. The vertical lines indicate the radial positions of the surfaces tangent to the rf limiter ( $\left.\mathrm{x}_{\mathrm{RF}}\right)$ and the FS ( $\left.\mathrm{x}_{\mathrm{FS}}\right)$.

Fig. 8 The rf enhancement of the radial plasma flux to the antenna, $\Gamma_{\mathrm{rf}} / \Gamma_{\mathrm{oh}}$, vs rf power in MW for 0 and $\pi$ phasing. The data points are inferred from the measured density profiles by a least-squares fit of the square-well CC model; the curves are the CC model predictions using parameters from the ANSAT sheath analysis. The closed circles and solid line denote $\pi$ phasing; the open circles and dashed line denote 0 phasing; the dotted line corresponds to 0 phasing with twice the calculated sheath voltage to indicate the range of uncertainty in the theoretical model.

Fig. 9 Theoretical predictions of $\Gamma_{\mathrm{rf}} / \Gamma_{\mathrm{oh}}$ vs $\mathrm{P}_{\mathrm{rf}}$ for 0 (dashed line), $\pi / 2$ (dotted line) and $\pi$ (solid line) phasings using the square-well model and the same parameters as in Fig. 8 except that the antenna-plasma separation has been reduced from $12 \mathrm{~mm}$ to $5 \mathrm{~mm}$. Note the large increase in phasing dependence as $\mathrm{P}_{\mathrm{rf}}$ increases and the convective cell broadens.

Fig. 10 Time histories of Ti line intensity (170 ̊ line intensity in arbitrary units) and $\mathrm{P}_{\text {rf }}$ for comparable shots with $\pi$ phasing (shot $\# 82576$, solid line) and $+\pi / 2$ phasing (shot \#82601, dashed line).

Fig. 11 History of $\mathrm{P}_{\text {rf }}$ (in MW at $3.3 \mathrm{~s}$ ) and Ti $170 \AA$ line intensity (in arbitrary units) at $1.0 \mathrm{~s}\left(\mathrm{Ti}_{10}\right), 2.1 \mathrm{~s}\left(\mathrm{Ti}_{21}\right)$, and $3.3 \mathrm{~s}\left(\mathrm{Ti}_{33}\right)$ for a sequence of shots comparing $\pi$ and $\pm \pi / 2$ phasings. $\mathrm{Ti}_{10}$ and $\mathrm{Ti}_{21}$ correspond to small and full-size ohmic plasmas, whereas $\mathrm{Ti}_{33}$ is measured during the rf pulse and correlates with rf power.

Fig. $12 \mathrm{Ti}$ line intensity $\mathrm{Ti}_{33}$ (in arbitrary units) vs rf power for the same sequence of shots as in Fig. 11. The antenna phasing is indicated by the symbols: $\pi$ (solid circles), $+\pi / 2$ (open squares), and $-\pi / 2$ (filled squares). 
Fig. 13 Ti concentration $\mathrm{f}_{\mathrm{Ti}}=\mathrm{n}_{\mathrm{Ti}} / \mathrm{n}_{\mathrm{e}}$, inferred from soft X-ray spectroscopy, for shots at several rf power levels and using different phasings: $\pi$ (solid circles), $+\pi / 2$ (open squares), and $-\pi / 2$ (filled squares). The values of $\mathrm{f}_{\mathrm{Ti}}$ are multiplied by $10^{4}$ for ease of display.

Fig. 14 (a) Calculated values of the Ti influx $\Phi_{\mathrm{FS}}$ at the FS versus rf power for those shots in Fig. 12 for which reflectometer data is available; (b) Calculated fraction T of Ti atoms from the FS which are directly transmitted into the core plasma. The meaning of the symbols is the same as in Fig. 12. For each shot, reflectometer data was employed to infer a plasma flux into the antenna, as described in the text.

Fig. 15 Comparison of the experimentally-measured Ti concentration with the theoretically-predicted concentration based on the direct influx from the FS. The solid line denotes agreement between the direct influx theory and experiment, and the arrows indicate the time sequence of the shots. The meaning of the symbols is the same as in Fig. 12. 


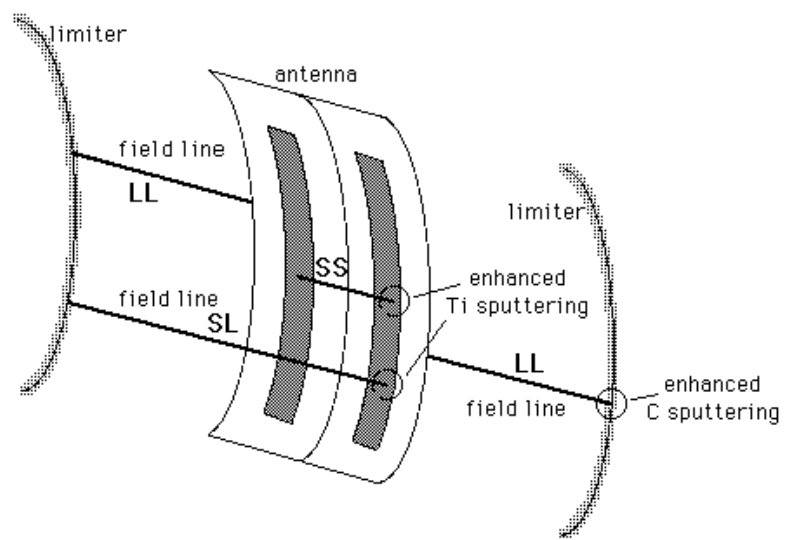

Fig. 1 


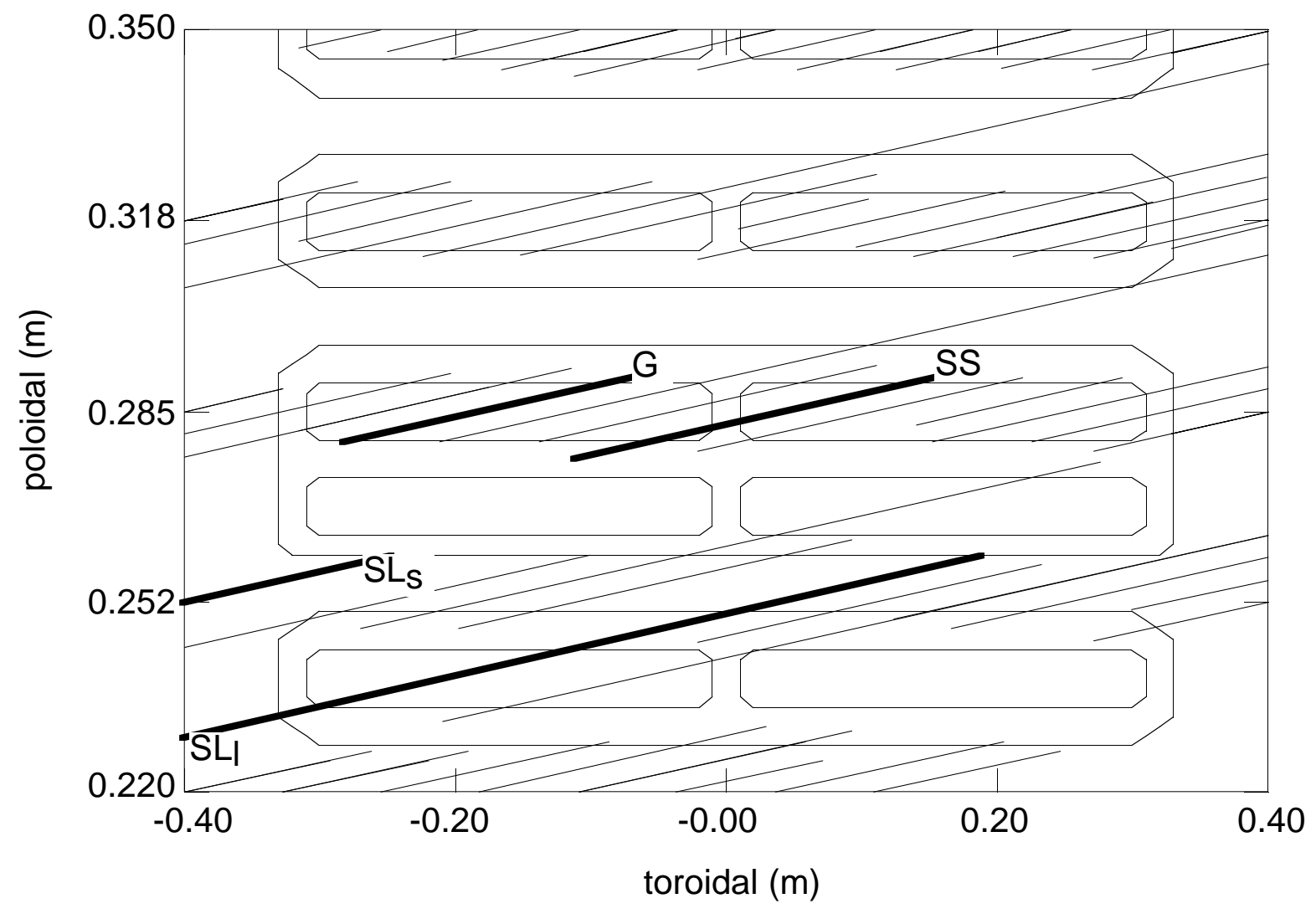

Fig. 2 

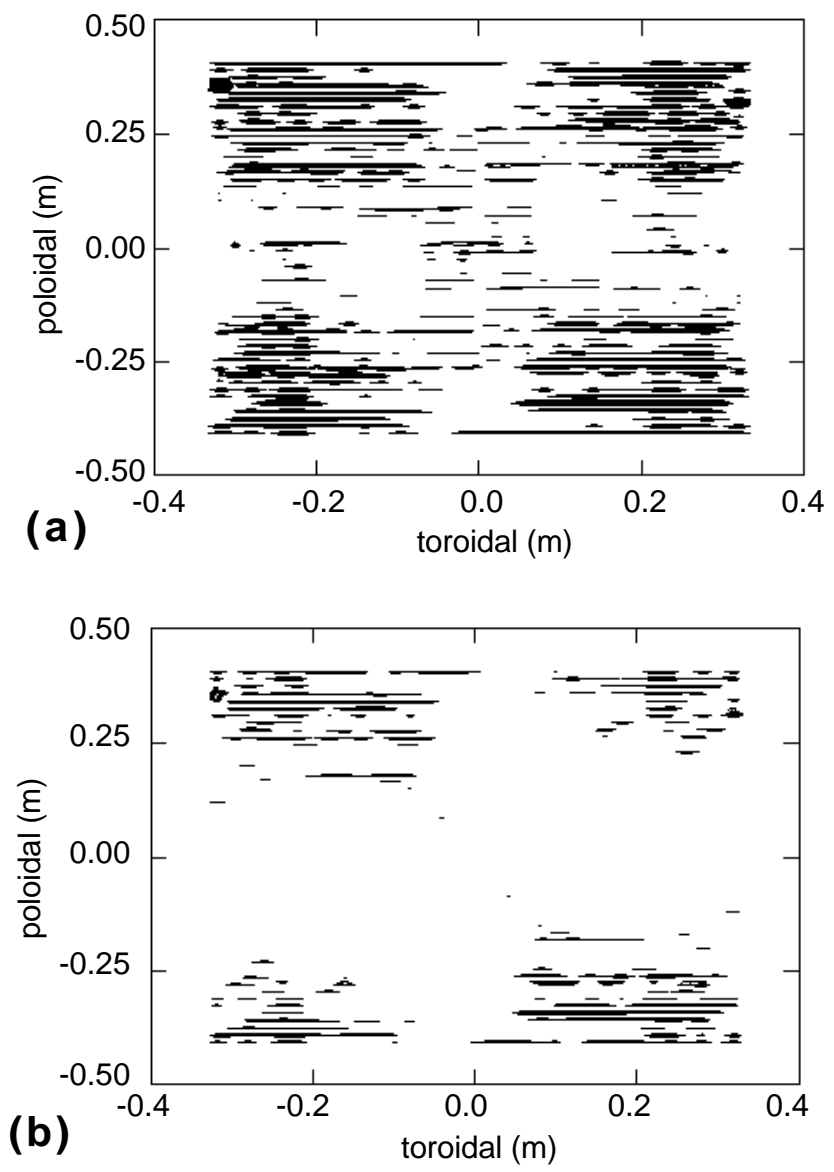

Fig. 3 

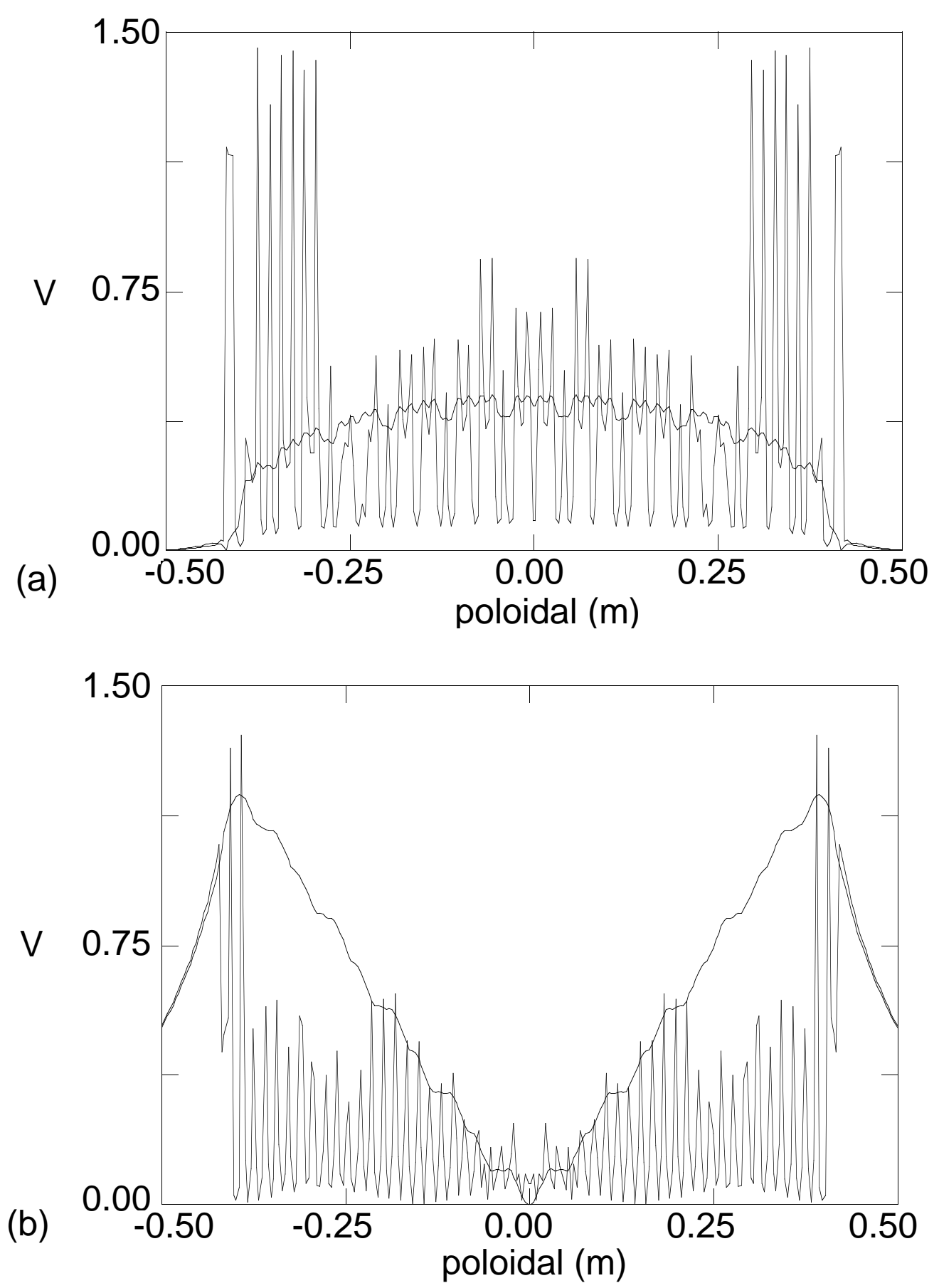

Fig. 4 

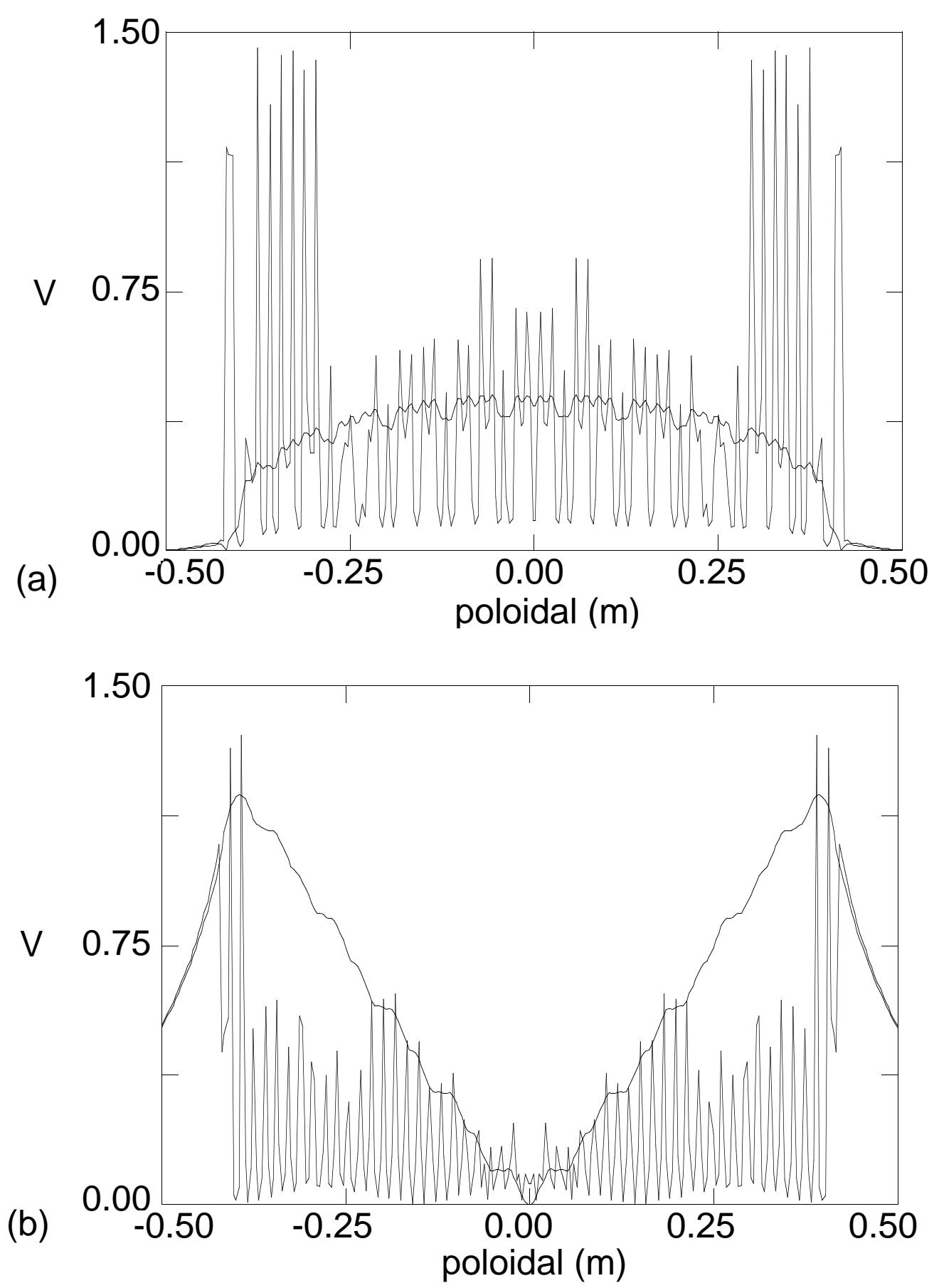

Fig. 4 

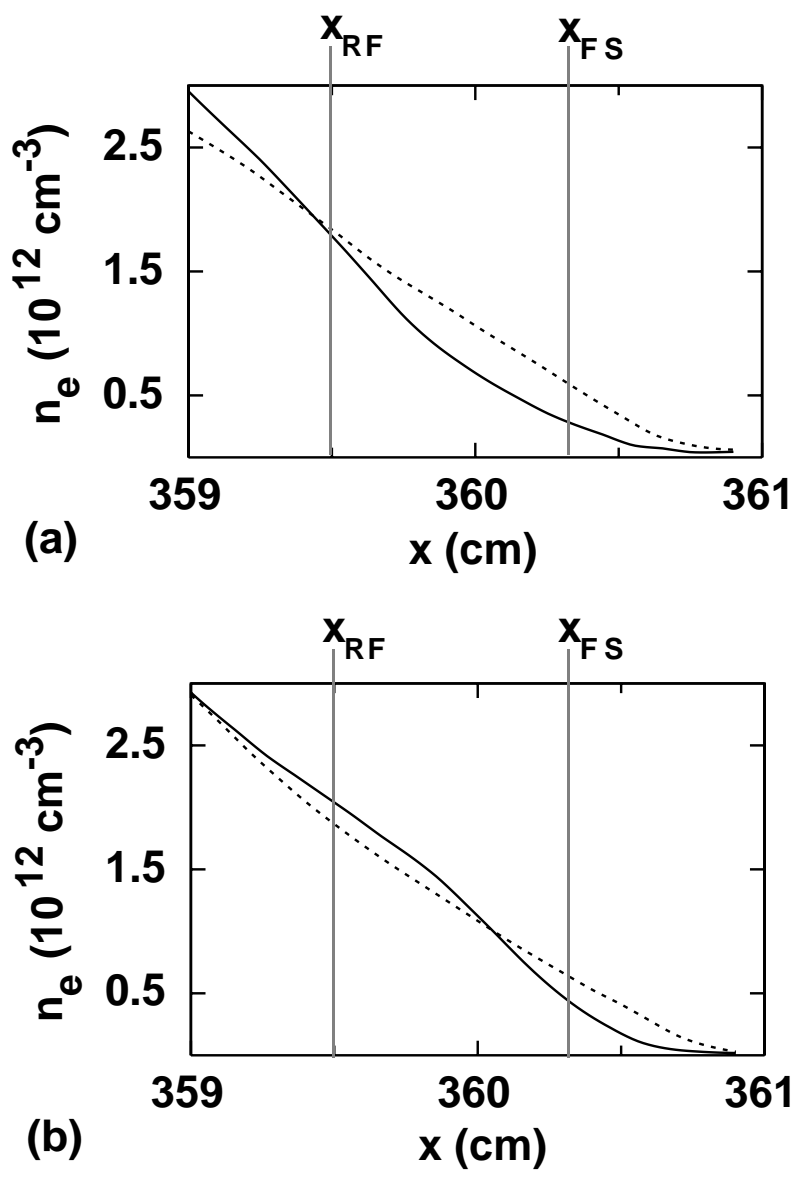

Fig. 5 


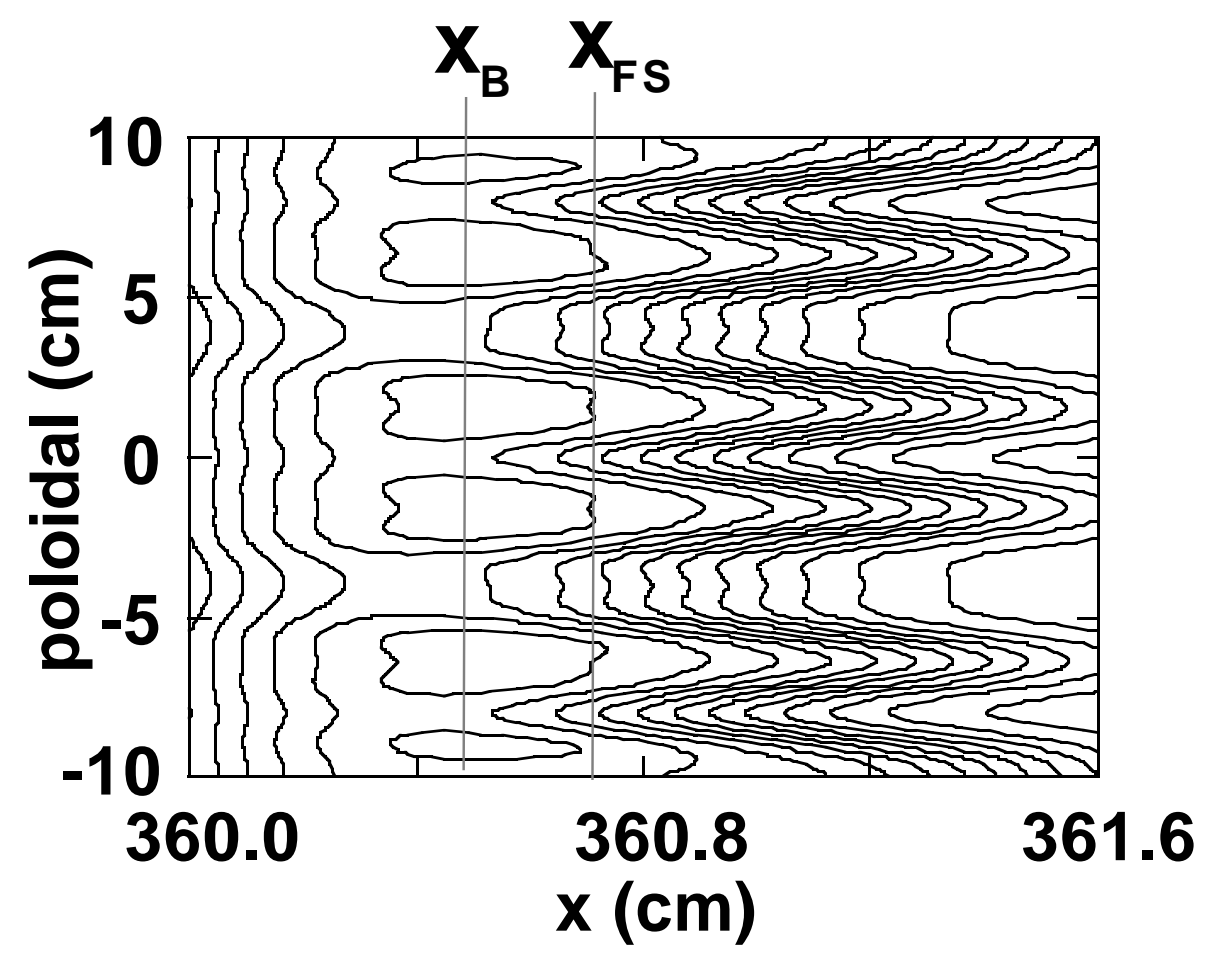

Fig. 6 


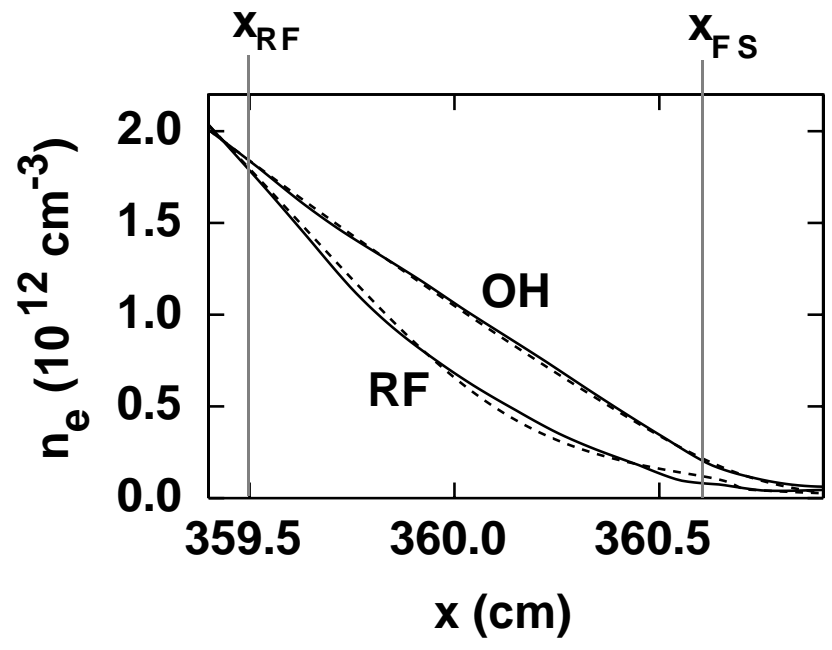

Fig. 7 


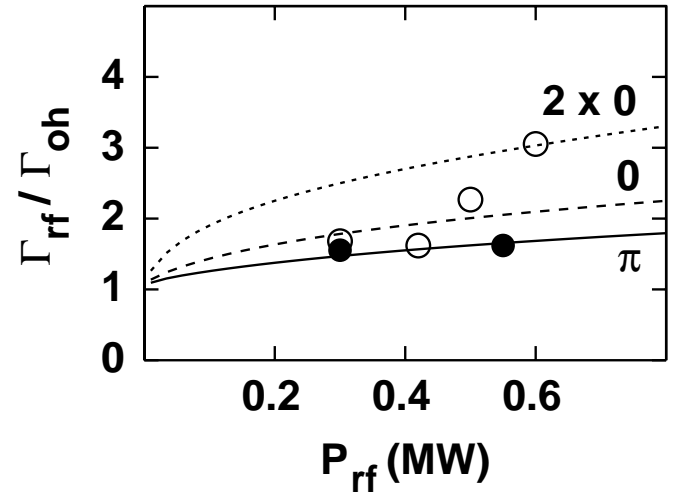

Fig. 8 


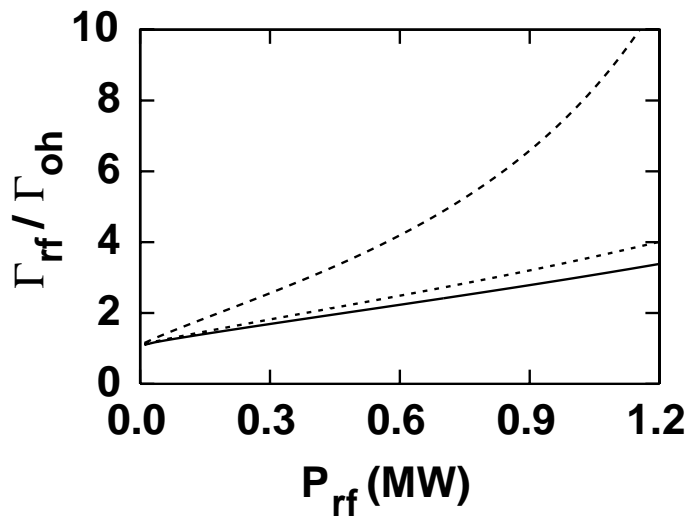

Fig. 9 

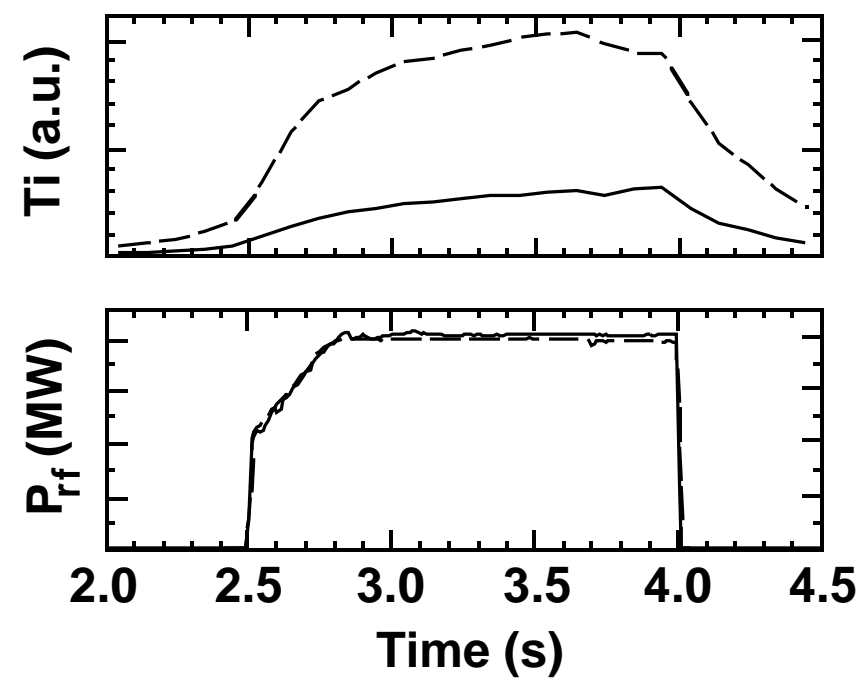

Fig. 10 

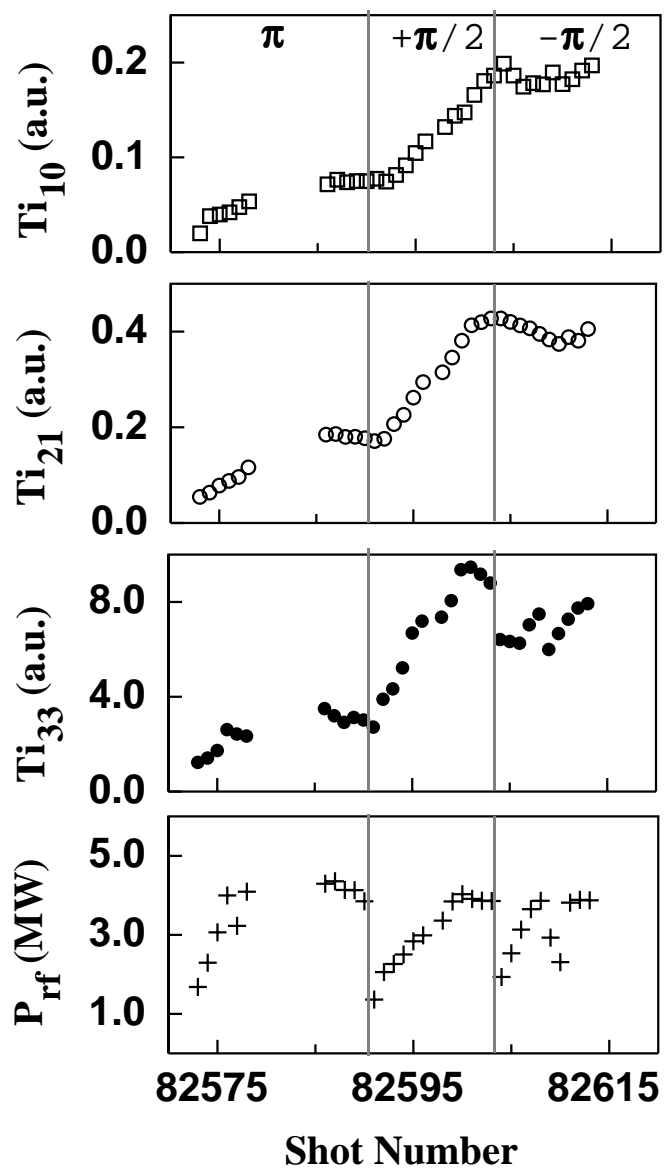

Fig. 11 


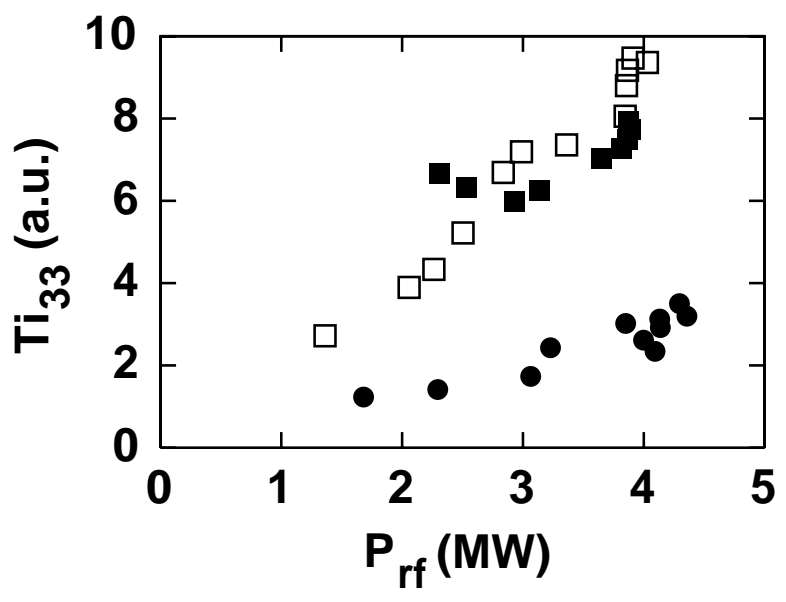

Fig. 12 


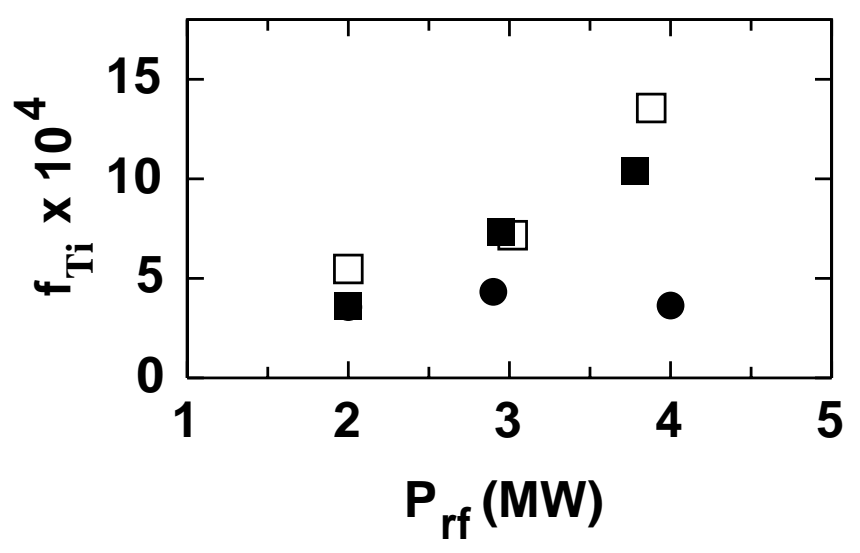

Fig. 13 

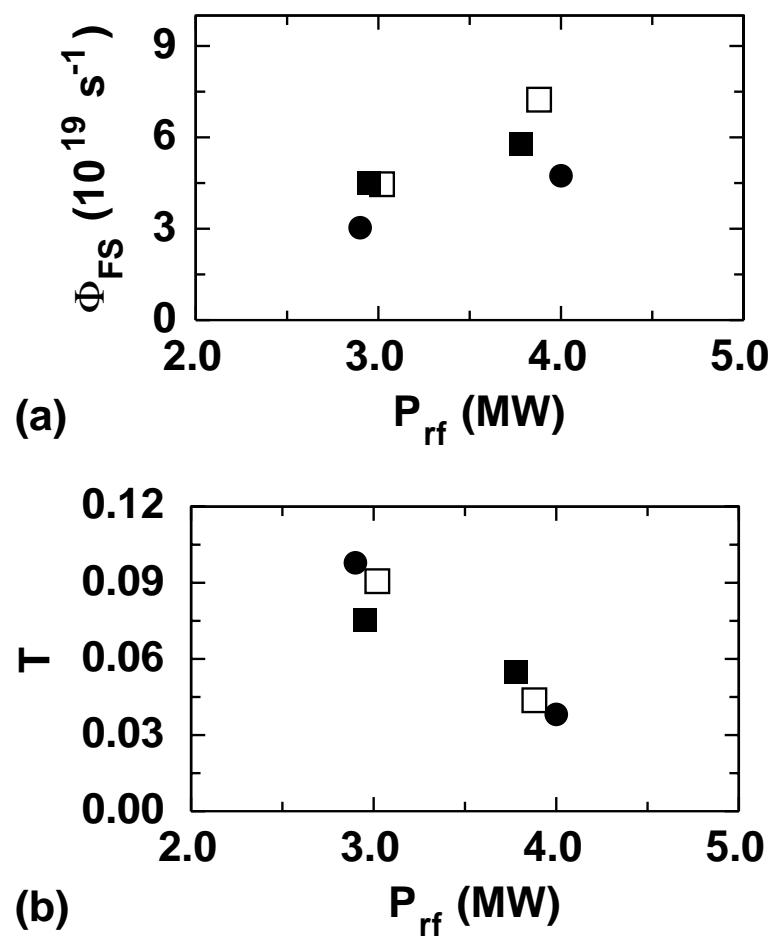

Fig. 14 


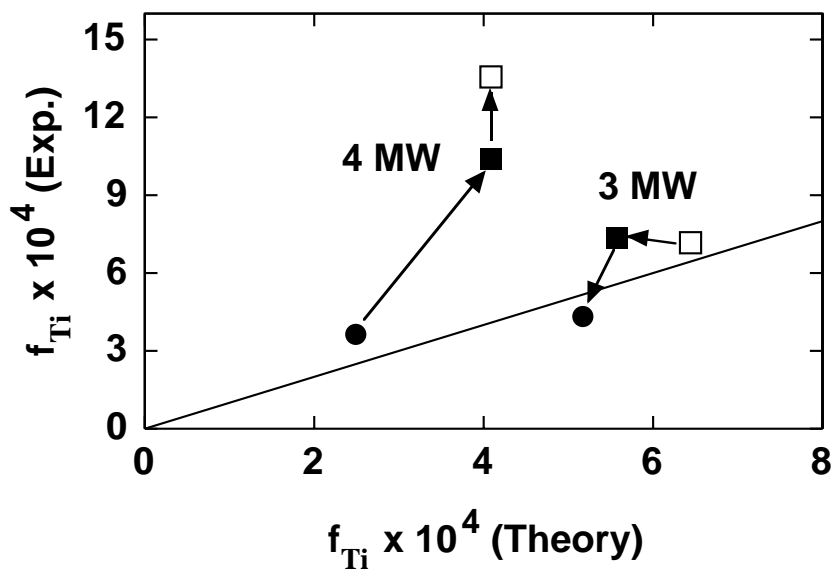

Fig. 15 